\title{
Structure and Energetics of Non-geostrophic Non-hydrostatic Baroclinic Instability Wave with and without Convective Heating
}

\author{
Wataru YANASE and Hiroshi NIINO \\ Ocean Research Institute, The University of Tokyo, Tokyo, Japan
}

(Manuscript received 10 March 2003, in final form 12 May 2004)

\begin{abstract}
Non-geostrophic non-hydrostatic baroclinic instability with and without convective heating is reexamined with an emphasis on the structure and energy budget of growing modes.

When the convective heating is absent, only a baroclinic instability mode has a positive growth rate for $R i=2$, where $R i$ is the Richardson number. The growth rate for a mode with meridional wavenumber $l(l>0)$ is proved to be exactly equal to that with $-l$. However, the energy budgets for the modes with $\pm l$ are found to be quite different: the mode with $l(>0)$ gains its eddy kinetic energy (EKE) from both eddy available potential energy (EAPE) and mean kinetic energy (MKE), while the mode with $-l$ gains EKE from EAPE, but loses it to MKE.

When the convective heating is included, at least two different growing modes are found to exist for $R i=2$ : One is a baroclinic instability mode (B-mode), modified by the convective heating, and the other a symmetric instability mode (S-mode) destabilized by the convective heating. The convective heating increases the growth rates of B-mode with $l(>0)$ more remarkably than that with $-l$. Energy budget analysis shows that the energy conversion between MKE and EKE is little affected by the convective heating, but that from EAPE to EKE increases more for the mode with $l$ than for that with $-l$, resulting in a larger growth rate for the mode with $l$. For the most unstable wavenumber, B-mode has an eastward tilt of the trough with increasing height in the lower layer and a cold air to the east of the surface trough. The thermodynamic analysis shows that this cold air is caused by adiabatic cooling at the low-levels due to the updraft driven by the convective heating at the mid- and upper-levels. The present results give a useful basis for understanding the dynamics of meso- $\alpha$-scale disturbances along the Baiu front and polar lows.
\end{abstract}

\section{Introduction}

Observational studies show that meso- $\alpha$-scale disturbances develop in certain environmental conditions in mid- and high-latitudes. These include "medium-scale or intermediate-scale disturbances" along the "Baiu front" around the Japan islands (Matsumoto et al. 1970; Ninomiya and Akiyama 1971; Yoshizumi 1977) and "polar lows" which develop over high-latitude

Corresponding author: Wataru Yanase, Ocean Research Institute, The University of Tokyo, 1-15-1 Minamidai, Nakano 164-8639, Tokyo, Japan.

E-mail: yanase@ori.u-tokyo.ac.jp

(C) 2004, Meteorological Society of Japan oceans (Harrold and Browning 1969; Reed and Duncan 1987; Hewson et al. 2000). These disturbances continue to attract the attention of meteorologists because their development mechanism has not been well understood yet.

One of the possible candidates for the development mechanism of such small disturbances is baroclinic instability that includes the effect of convective heating. A number of meteorologists have investigated this mechanism using "two-dimensional" quasi-geostrophic equations that include a parameterization of condensational heating (Mak 1982; Sardie and Warner 1983; Moorthi and Arakawa 1985; Mak 1994). Mak (1982) adopted a CISK-type parameter- 
ization in which the convective heating is related to the low-level vertical velocity, and revealed that the convective heating increases the growth rate and decreases the horizontal scale of baroclinic instability wave. It is also pointed out that the convective heating destabilizes several additional modes the structures of which are different from those of the ordinary baroclinic instability waves (Moorthi and Arakawa 1985; Mak 1994). Moorthi and Arakawa (1985) demonstrated that strong convective heating within an easterly shear causes the Green mode in which the energy generation due to the convective heating dominates the baroclinic energy conversion. Mak (1994) adopted a CISK-type parameterization with the top-hat heating profile, and showed that the potential vorticity generation at the discontinuity of convective heating causes the destabilization of additional modes. These previous studies revealed that the effects of convective heating depends on the amount and profile of the heating and the relation between heating and vertical velocity (Sardie and Warner 1983; Moorthi and Arakawa 1985).

For meso- $\alpha$-scale disturbances, for which the Richardson number $R i$ of the environmental atmosphere is of the order unity, however, quasi-geostrophic equations are not suitable for describing the behavior: in a non-geostrophic system, an energy conversion between eddy kinetic energy and mean kinetic energy of vertical wind shear appears, and starts to affect the energy budget of the disturbances. This also causes additional instability modes. In fact, Stone (1966) showed that, for a nongeostrophic adiabatic atmosphere, the symmetric instability wave appears in addition to baroclinic instability mode for $R i<1$.

When the convective heating is included in the non-geostrophic system, baroclinic instability waves are deformed even for $R i>1$ (Tokioka 1973; Gambo 1976). Tokioka (1973) (hereafter referred to as T73) studied a nongeostrophic and non-hydrostatic stability of the three-dimensional Eady model with a CISKtype convective heating. He found that the zonal and meridional scales of the fastest growing mode of the baroclinic instability for $R i=2$ become smaller in the presence of convective heating, which is similar to the results of quasi-geostrophic system mentioned above. He speculated that these results may explain why the disturbances with smaller horizontal scale develop along the Baiu front. T73 has also shown that the disturbance with positive meridional wavenumber has a larger growth rate than that with negative meridional wavenumber, and the structure of the most unstable wave resembles that of some disturbances observed along the Baiu front: the trough axis tilts eastward with increasing height and a cold air exists on the east side of the trough in the lower atmosphere (e.g., Ninomiya and Akiyama 1971; Yoshizumi 1977). However, the cause for the asymmetry with respect to the meridional wavenumber and the eastward tilt of the trough have not been clarified so far.

Recent development of computer technology makes cloud-resolving simulation of polar lows (e.g., Yanase et al. 2002), hurricanes (e.g., Romine and Wilhelmson 2002) and medium-scale disturbances more and more feasible. When the results of these sophisticated numerical simulations are interpreted, however, the basic understanding of the dynamics of non-geostrophic baroclinic waves with convective heating is indispensable. T73 gave important steps to such an understanding. As mentioned above, however, it still possesses unresolved questions to be clarified. In the present study, we will thus re-examine the structure and energy budget of the non-geostrophic non-hydrostatic baroclinic instability with and without convective heating (Tokioka 1970; T73) in order to clarify the following point: (1) Why does baroclinic instability mode with convective heating have a structure in which the trough slopes eastward with increasing height in the lower layer? (2) Why does the baroclinic instability mode with convective heating have a larger growth rate for positive meridional wavenumber than for negative wavenumber? (3) What is the energy budget for the unstable modes? (4) T73 examined only B-mode modified by the convective heating by using a shooting method. In the present paper, a matrix method is used to explore the possibility if there are other modes in this system.

The problem is formulated in Section 2. The results are described in Section 3, and detailed discussion including physical interpretation of the results are presented in Section 4. Finally, the results are summarized in Section 5. Note 
that Appendix is provided to clarify the relationship between the non-geostrophic nonhydrostatic system and the quasi-geostrophic system.

\section{Formulation of the problem}

\subsection{Model}

The model considered in this paper is exactly the same as that of Tokioka (1973) who considered a linear non-geostrophic non-hydrostatic stability of a baroclinic flow with the effect of CISK-type convective heating. The basic state is the same as the Eady problem (Eady 1949). The basic zonal flow is uniform in the north $(y)$ direction, and has a constant vertical shear $\Lambda(>0)$ in the range $0 \leq z \leq z_{0}$ :

$$
\bar{u}=\Lambda\left(z-z_{0} / 2\right) \text {, }
$$

where $z_{0}$ is taken to be $5 \mathrm{~km}$ in this study. The basic potential temperature field has a uniform static stability, and satisfies the thermal wind balance:

$$
\bar{\theta}=-f \Lambda y / g \alpha+S z,
$$

where $\alpha$ is the coefficient of thermal expansion, $f$ the Coriolis parameter, $g$ the gravity acceleration, and $S$ the constant vertical gradient of the basic potential temperature field. Using these parameters, the Richardson number is given as $R i=g \alpha S / \Lambda^{2}$. Assuming infinitesimally small perturbations of the form $\psi=$ $\hat{\psi}(z) \exp (i k x+i l y-i \sigma t)$ superposed on the basic field, we obtain linearized perturbation equations under the Boussinesq approximation:

$$
\begin{aligned}
& i(k \bar{u}-\sigma) \hat{u}+\Lambda \hat{w}-f \hat{v}=-i k \hat{\phi}, \\
& i(k \bar{u}-\sigma) \hat{v}+f \hat{u}=-i l \hat{\phi}, \\
& i(k \bar{u}-\sigma) \hat{w}-g \alpha \hat{\theta}=-d \hat{\phi} / d z, \\
& i(k \bar{u}-\sigma) \hat{\theta}-f \Lambda \hat{v} / g \alpha+S \hat{w}=X_{\theta}, \\
& i k \hat{u}+i l \hat{v}+d \hat{w} / d z=0 .
\end{aligned}
$$

The variables $\hat{u}, \hat{v}$ and $\hat{w}$ are the perturbation velocity components in the zonal, meridional and vertical directions, respectively, $\hat{\theta}$ the perturbation potential temperature, and $\hat{\phi}$ the perturbation geopotential. In the thermodynamic equation (6), $X_{\theta}$ indicates the heating rate due to cumulus convection. As in T73, the heating rate is assumed to be related to the low level updraft as follows:

$$
X_{\theta}=\operatorname{Sh}(z) \hat{w}^{*},
$$

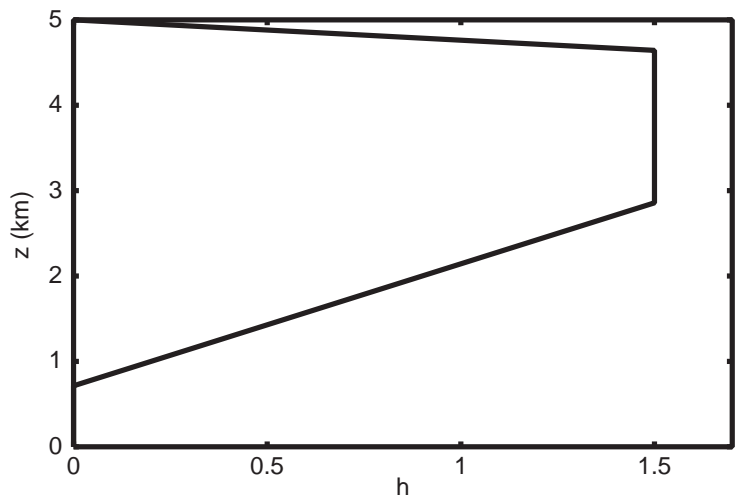

Fig. 1. Vertical profile of $h(z)$ in Eq. (8). $h(z)$ is given by linear functions of $z$ between 0.71 and $2.66 \mathrm{~km}$ and between 4.65 and $5.00 \mathrm{~km}$, and is equal to 1.5 between 2.66 and $4.65 \mathrm{~km}$.

where $h$ is the vertical profile of heating rate as shown in Fig. 1 and $\hat{w}^{*}$ the vertical wind velocity at the low level $\left(z=z^{*} \equiv 0.71 \mathrm{~km}\right)$. We refer to the case in which $X_{\theta}$ is given by Eq. (8) as the "moist" model, and to that in which $X_{\theta}$ is zero as the "dry" model. The latter corresponds to the ordinary baroclinic instability problem except that the non-geostrophic and non-hydrostatic effects are considered. In order to keep the model as simple as possible, we exclude the effect of the momentum transport by the cumulus convection and the surface friction which are included in T73. In Appendix, we have summarized the features of the nongeostrophic non-hydrostatic system in nondimensional form.

The boundary conditions are $\hat{w}=0$ at the top and bottom boundaries $\left(z=0, z_{0}\right)$. Equations (3)-(7) are discretized for 70 vertical layers using a finite difference method: the variables $\hat{u}, \hat{v}, \hat{\phi}$ and $\hat{\theta}$ are given at the center of each layer (i.e., 70 levels), while $\hat{w}$ is given at boundaries between the layers (i.e., 71 levels). The matrix method is used to solve the eigenvalue problem and to obtain all the eigenvalues. Note that, in T73, the shooting method was used to find the eigenvalues only for one baroclinic instability mode.

\subsection{Energetics}

Though the formulation of the energy conversions among the perturbation and the mean flow are well known, we will briefly review them for reader's convenience. Time rate of 
the horizontally-averaged eddy kinetic energy $K^{\prime}\left(\equiv \frac{\overline{u^{2}}+\overline{v^{2}}+\overline{w^{2}}}{2}\right)$ at each level is given by

$$
\frac{\partial K^{\prime}}{\partial t}=-\Lambda \overline{u w}+g \alpha \overline{w \theta}-\frac{d \overline{w \phi}}{d z},
$$

where the overbar denotes a horizontal average, and the prime a quantity associated with the perturbation. The first term $-\Lambda \overline{u w}$ in the right-hand side (RHS) is the conversion from mean kinetic energy $\bar{K}$ to eddy kinetic energy $K^{\prime}$, and the second term $g \alpha \overline{w \theta}$ in RHS the conversion from eddy available potential energy $P^{\prime}$ to eddy kinetic energy. Hereafter, the former is denoted by $\left[\bar{K}, K^{\prime}\right]$ and the latter by $\left[P^{\prime}, K^{\prime}\right]$. Note that $\left[\bar{K}, K^{\prime}\right]$ is absent in a quasigeostrophic system (see Appendix). We are not concerned with the third term $-\frac{d \overline{w \phi}}{d z}$ in RHS because it disappears when the equation is integrated vertically over the whole domain.

The equation for the horizontally-averaged eddy available potential energy $P^{\prime}\left(\equiv \frac{g \alpha \theta^{2}}{2 S}\right)$ is

$$
\frac{\partial P^{\prime}}{\partial t}=\frac{f \Lambda}{S} \overline{v \theta}+g \alpha h \overline{w^{*} \theta}-g \alpha \overline{w \theta} .
$$

The first term $\frac{f \Lambda}{S} \overline{v \theta}$ in RHS is the conversion from mean available potential energy $\bar{P}$ to eddy available potential energy, and the second term $g \alpha h \overline{w^{*} \theta}$ in RHS the production of eddy available potential energy by convective heating. These terms are denoted by $\left[\bar{P}, P^{\prime}\right]$ and $\left[Q, P^{\prime}\right]$, respectively. The third term $-g \alpha \overline{w \theta}$ in RHS is the conversion from eddy kinetic energy to eddy available potential energy which is just equal to the second term in RHS of Eq. (9) except for the sign.

\section{Results}

In what follows, we will concentrate on the case $R i=2$ in which non-geostrophic effects are expected to operate remarkably. This case of small Richardson number has been most extensively examined by T73 because the convective heating greatly modifies the structures of the baroclinic instability waves. The dimensional parameters used for the present calculation are $f=10^{-4} \mathrm{sec}^{-1}, g \alpha S=0.5 \times 10^{-4} \mathrm{sec}^{-2}$ and $\Lambda=0.5 \times 10^{-2} \mathrm{sec}^{-1}$.

\subsection{Unstable modes and their growth rates}

Figure 2 shows the maximum growth rate as a function of the zonal wavenumber $k$ and (a) dry

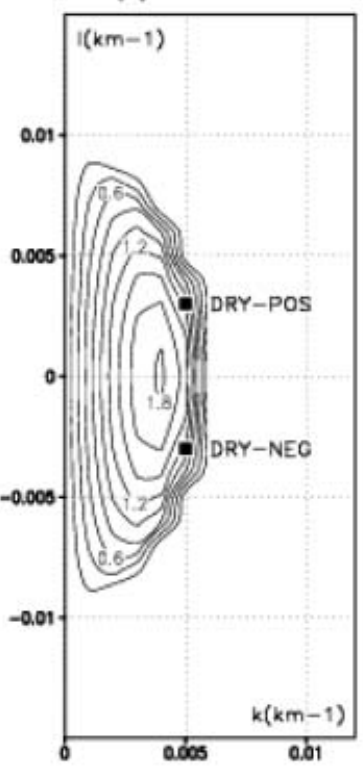

(b) moist

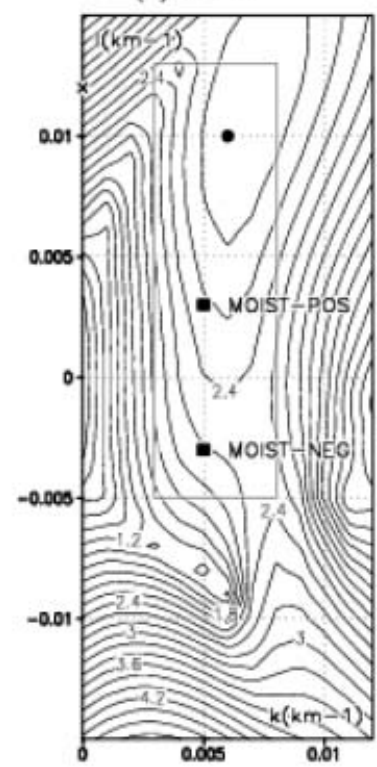

Fig. 2. Growth rate curve in the zonal and meridional wavenumber plane (a) for the dry experiment and (b) for the moist experiment when $R i=2.0$. The contour interval is $0.2 \times 10^{-5} \mathrm{~s}^{-1}$. The inner rectangle in the panel (b) shows the wavenumber domain analyzed by T73. The closed circle, closed squares, cross and letters denote the wavenumbers and their names which are analyzed in the present study.

meridional wavenumber $l$ for dry (Fig. 2a) and moist (Fig. 2b) models. The peak of the growth rate for the dry model occurs at $k \approx 0.004 \mathrm{~km}^{-1}$ and $l=0$. As is proved in Appendix, the growth rate is exactly symmetric with respect to the $k$ axis.

The growth rate for the moist model (Fig. 2b) shows a very complicated distribution because several additional modes seem to be destabilized by the convective heating. The rectangle in Fig. $2 \mathrm{~b}$ shows the domain of $(k, l)$ that is examined by T73. In this domain, the most unstable mode seems to consist of only the mode obtained by T73 (hereafter referred to as "Bmode"). It has a peak growth rate at around $k=0.007 \mathrm{~km}^{-1}$ and $l=0.013 \mathrm{~km}^{-1}$. One of the notable features of B-mode is that its growth rate is asymmetric with respect to the $k$ axis, and has a maximum for a positive meridional 
wavenumber. The B-mode is considered to be a baroclinic instability mode modified by the effect of the convective heating, because it has been confirmed that the position of the peak growth rate of the dry baroclinic instability (Fig. 2a) shifts continuously to that of B-mode (Fig. 2b), if the peak heating rate $h(z)$ between 2.68 and $4.46 \mathrm{~km}$ is gradually increased from 0 to 1.5 (see Fig. 1).

In the following subsections, we will first examine the structure of B-mode at the most unstable wavenumber, and then proceed to analyze the reason why B-mode exhibits the asymmetry with respect to the $k$ axis. Also examined is the additional unstable mode that appears outside of the rectangle in Fig. $2 \mathrm{~b}$, and was not studied by T73.

\subsection{Structure of the most unstable B-mode}

Figure 3 shows the zonal-vertical cross section of $u, v, w, \phi, \theta$ and the terms in the thermodynamic equation (6) for the most unstable Bmode. This confirms the results of T73 except that T73 did not give the cross sections of $u, v$ and the terms of the thermodynamic equation. The meridional wind does not satisfy the geostrophic balance with the geopotential field near the bottom and top boundaries (Figs. 3b and $3 \mathrm{~d}$ ). The strongest meridional wind does not occur at the maximum or minimum zonal gradient of the geopotential; rather, it occurs near the maximum or minimum geopotential. The zonal wind is nearly proportional to the zonal gradient of the geopotential near the bottom and top boundaries. Like dry baroclinic waves, $v$ and $w$ have a positive correlation particularly in the mid-level. Most notable difference in the structure of B-mode from that of the dry baroclinic waves is that its trough axis tilts eastward in the lower layer (Fig. 3d). Such a structure has been also observed within meso$\alpha$-scale disturbances in the Baiu front (e.g., Ninomiya and Akiyama 1971; Yoshizumi 1977). The potential temperature and geopotential fields approximately satisfy the hydrostatic balance, and a cold air exists on the east side of the trough in the lower layer (around $210^{\circ}<x<360^{\circ}$ and $z<2 \mathrm{~km}$ in Fig. 3e). Note that Fig. 3 may be also regarded as the meridional-vertical cross sections of the variables because the variables are proportional to $\exp (i k x+i l y-i \sigma t)$.
It looks curious that the cold air exists on the east side of the trough at low levels, because, in the case of dry baroclinic waves, warm air exists just to the east of the trough due to the northward advection of warm air. In order to understand the cause of the cold air to the east of the trough at low levels, we have investigated the balance among the terms in the thermodynamic equation (Figs. 3f-j). Figure 3f shows the tendency of the potential temperature $(-i \sigma \theta)$, which shows a nearly similar pattern to that of the potential temperature (Fig. $3 e$ ). This tendency is determined by the sum of the four terms in the thermodynamic equation (see Eq. (6)): the zonal advection (-ikU $\theta$; Fig. $3 \mathrm{~g})$, the meridional advection ( $f \Lambda v / g \alpha$; Fig. $3 \mathrm{~h})$, the vertical advection $(-S w$; Fig. $3 \mathrm{i})$ and the convective heating $\left(X_{\theta} ;\right.$ Fig. $\left.3 \mathrm{j}\right)$. It is clearly seen that the vertical advection dominates where the cold air on the east side of the trough at the low levels occurs. In other words, the adiabatic cooling within the strong updraft is dominant there. Note that in the dry baroclinic wave, the contribution of the vertical advection is smaller than that of the meridional advection so that warm air exists just to the east of the low-level trough. The strong updraft on the east side of the trough for the moist wave is caused by the large condensational heating in the upper layer.

Now, let us look at the energy cycle for the most unstable B-mode. Figure $4 \mathrm{a}$ shows the vertical profiles of the energy conversion terms normalized by the vertically-integrated $K^{\prime}$. These profiles nearly coincide with those presented in Fig. 6 of T73: The conversion $\left[\bar{P}, P^{\prime}\right]$ is largest above $4 \mathrm{~km}$ above ground level (AGL), and is negative between 0.5 and $2 \mathrm{~km} \mathrm{AGL.}$ The conversion $\left[Q, P^{\prime}\right]$ is small below $2 \mathrm{~km}$ AGL, but has a maximum at about $3 \mathrm{~km}$ AGL. Different from the Eady problem, the present non-geostrophic problem has a significant conversion $\left[\bar{K}, K^{\prime}\right]$ throughout the atmosphere with a maximum at about $2 \mathrm{~km}$ AGL. The conversion $\left[P^{\prime}, K^{\prime}\right]$ has a peak of similar magnitude to that of $\left[\bar{K}, K^{\prime}\right]$ between 3 and $4 \mathrm{~km} \mathrm{AGL,} \mathrm{but}$ becomes negative below $2 \mathrm{~km}$ AGL.

Figure $4 \mathrm{~b}$ shows the block diagram of the energy cycle, where the energy conversion terms are presented after a vertical integration. It is seen that the production of $K^{\prime}$ by $\left[\bar{K}, K^{\prime}\right]$ is approximately twice as large as that by $\left[P^{\prime}, K^{\prime}\right]$. 
(a) $u$

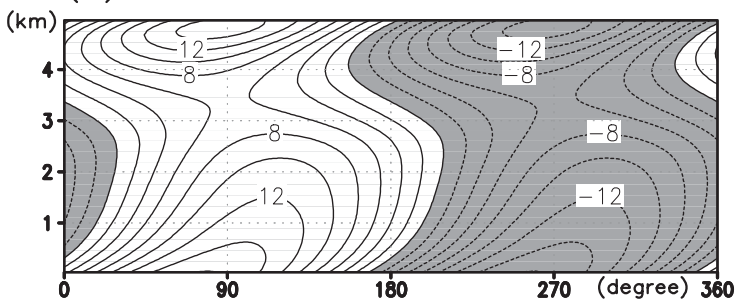

(c) $\mathrm{w}$

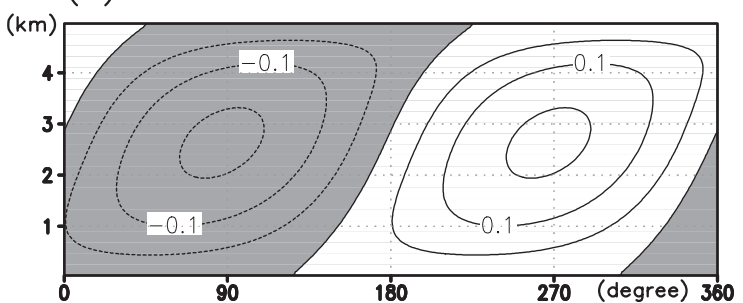

(e) $\theta$

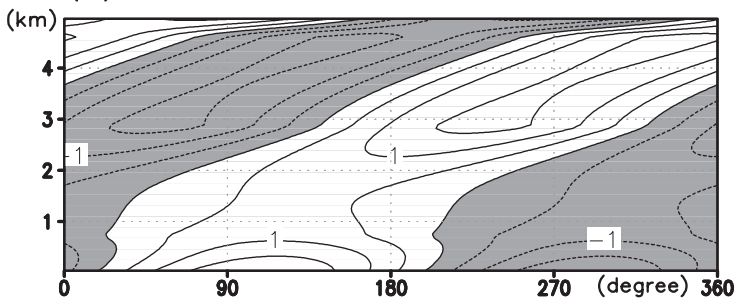

(g) zonal advection of $\theta$

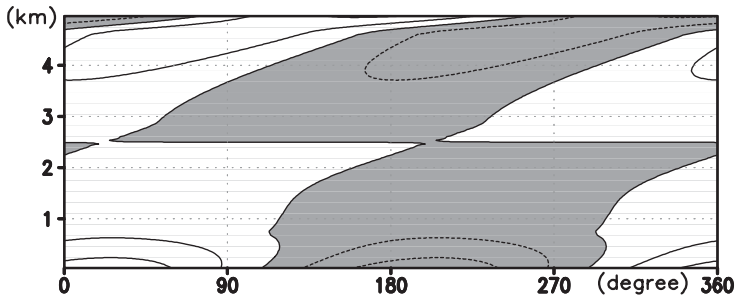

(i) vertical advection of $\theta$

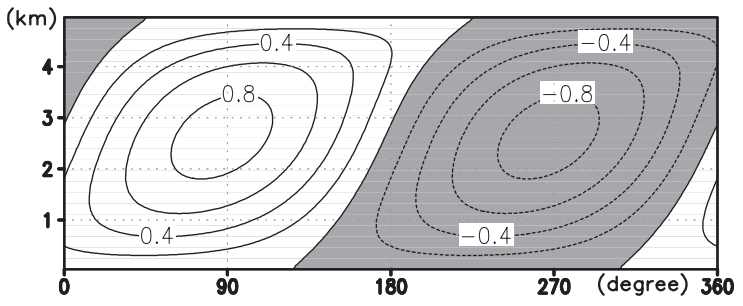

(b) $v$

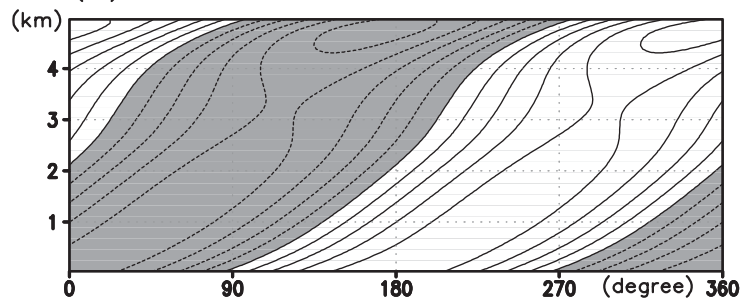

(d) $\phi$

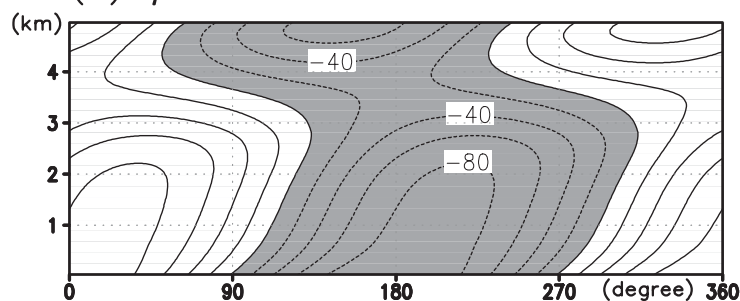

(f) tendency of $\theta$

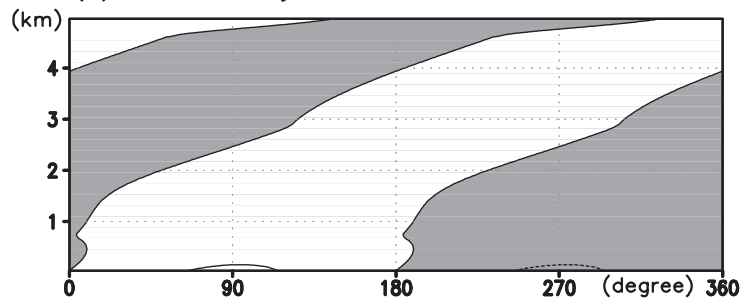

(h) meridional advection of $\theta$

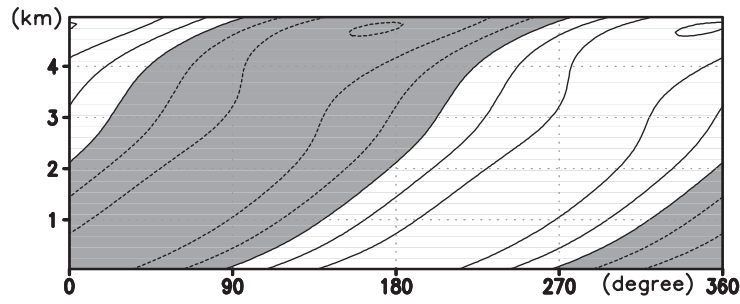

(j) convective heating

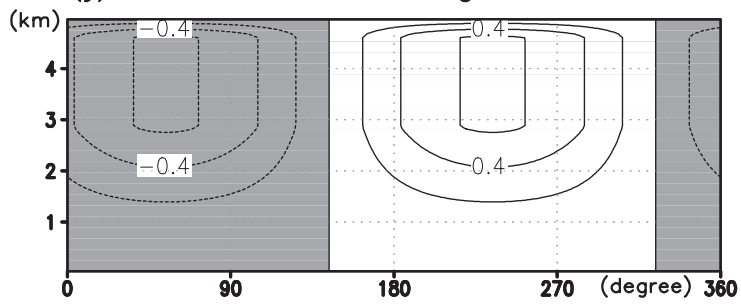

Fig. 3. Zonal-vertical cross section of the B-mode wave at the most unstable wavenumber $\left(k=0.006 \mathrm{~km}^{-1}, l=0.01 \mathrm{~km}^{-1}\right.$ ). (a) $u$ (contour interval (c.i.) is $2 \mathrm{~ms}^{-1}$ ); (b) $v$ (c.i. $=2 \mathrm{~ms}^{-1}$ ); (c) $w$ $\left(\right.$ c.i. $\left.=0.05 \mathrm{~ms}^{-1}\right)$; (d) $\phi\left(\right.$ c.i. $\left.=20 \mathrm{~m}^{2} \mathrm{~s}^{-2}\right)$; (e) $\theta(\mathrm{c} . \mathrm{i} .=0.5 \mathrm{~K}) ;(\mathrm{f})-(\mathrm{j})$ terms in the thermodynamic equation (c.i. $\left.=0.2 \mathrm{Khr}^{-1}\right)$ : (f) tendency $(-i \sigma \theta)$; $(\mathrm{j})$ zonal advection $(-i k \bar{u} \theta)$; $(\mathrm{h})$ meridional advection $(f \Lambda v / g \alpha)$; (i) vertical advection $(-S w)$; (j) convective heating $\left(X_{\theta}\right)$. The amplitude and phase of the wave are set so that the minimum geopotential of $-100 \mathrm{~m}^{2} \mathrm{~s}^{-2}$ at the lowest level occurs at 180 degrees in the zonal direction. The shaded regions indicate the negative values. 


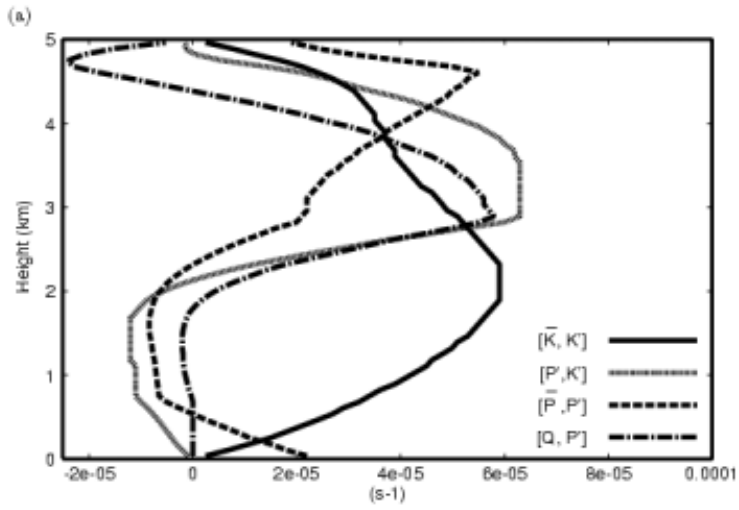

(b)

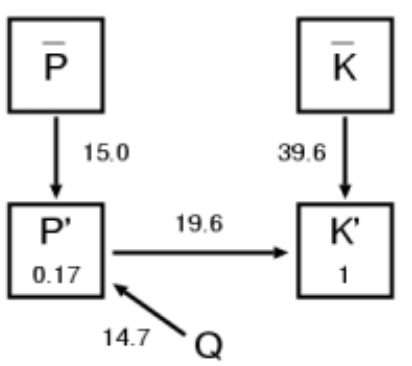

Fig. 4. Energy cycle for B-mode for $k=0.006 \mathrm{~km}^{-1}$ and $l=0.01 \mathrm{~km}^{-1}$. The values are normalized by the verticallyintegrated $K^{\prime}$. (a) Vertical profile of energy conversions. (b) Block diagram of the energy cycle. The numerals in the boxes of $K^{\prime}$ and $P^{\prime}$ indicate the energy and those with the arrows the energy conversion rates in the unit of $10^{-6} \mathrm{~s}^{-1}$, where the energy conversion rates are obtained by integrating vertically the energy conversions as shown in (a).

The ratio of $P^{\prime}$ to $K^{\prime}$ is 0.17 and is much smaller than unity. The ratio of $\left[\bar{K}, K^{\prime}\right]+\left[P^{\prime}, K^{\prime}\right]$ to $K^{\prime}$ and that of $\left[\bar{P}, P^{\prime}\right]+\left[Q, P^{\prime}\right]-\left[P^{\prime}, K^{\prime}\right]$ to $P^{\prime}$ are both $59.2 \times 10^{-6} \mathrm{~s}^{-1}$, which is exactly twice as large as the growth rate $\left(\sigma_{i} \approx 29.6 \times 10^{-6} \mathrm{~s}^{-1}\right)$ of this wave (see Fig. 2) as is expected from its exponentially growing behavior.

\subsection{Dependence of baroclinic instability mode on convective heating and meridional wavenumber}

In the previous subsections, we have briefly reviewed the results of T73. One of the questions that were not clarified by T73 is why the growth rate plotted on the horizontal wavenumber plane is asymmetric with respect to the $k$ axis for the moist experiment. In order to answer this question, we will make a comparative study on four types of waves which are denoted by the solid squares in Figs. $2 \mathrm{a}$ and $2 \mathrm{~b}$. These waves are selected to have the same zonal wavenumber $\left(k=0.005 \mathrm{~km}^{-1}\right)$ and the same absolute value of the meridional wavenumber $\left(|l|=0.003 \mathrm{~km}^{-1}\right)$. The waves with $l=$ $0.003 \mathrm{~km}^{-1}$ and $-0.003 \mathrm{~km}^{-1}$ for the dry model are called DRY-POS and DRY-NEG waves, respectively, while those for the moist model MOIST-POS and MOIST-NEG waves, respectively. We have confirmed that MOIST-POS and MOIST-NEG waves belong to the same mode by checking the absence of the other modes that have similar growth rates. All the four waves have positive growth rates.

\section{a. Dry waves}

First, let us look at the results of the dry model. Figures 5a and 5b show the block diagrams of the energy cycle for DRY-POS and DRY-NEG waves, respectively. These two waves are found to have quite different energetics, although they have exactly the same growth rate (see Fig. 2a): For DRY-POS wave, $\left[\bar{K}, K^{\prime}\right]$ and $\left[P^{\prime}, K^{\prime}\right]$ are nearly of the same magnitude. For DRY-NEG wave, on the other hand, $\left[\bar{K}, K^{\prime}\right]$ is negative and $\left[P^{\prime}, K^{\prime}\right]$ is larger than that of DRY-POS wave. The sum of $\left[\bar{K}, K^{\prime}\right]$ and $\left[P^{\prime}, K^{\prime}\right]$ for both waves, however, have the same magnitude $\sim 22.8 \times 10^{-6} \mathrm{~s}^{-1}$, resulting in the same growth rate for both waves.

The difference in the energy cycle between DRY-POS and DRY-NEG waves is closely related to the difference of the structure between the two waves. Figures 6 and 7 show the zonalvertical cross sections of the several variables and terms in the thermodynamic equation for DRY-POS and DRY-NEG waves, respectively. For both waves, the correlations between $v$ and $w$ are positive on the whole. The correlations between $u$ and $w$ for the two waves, however, have the different signs: For DRY-POS wave, the correlation between $u$ and $w$ is negative, which explains the positive value of $\left[\bar{K}, K^{\prime}\right]$. For DRY-NEG wave, on the other hand, the correlation is positive, which results in the negative value of $\left[\bar{K}, K^{\prime}\right]$.

At the mid-level, the correlations between $w$ and $\theta$ is high for both waves. Note that this feature also applies to the other waves in the 
(a)

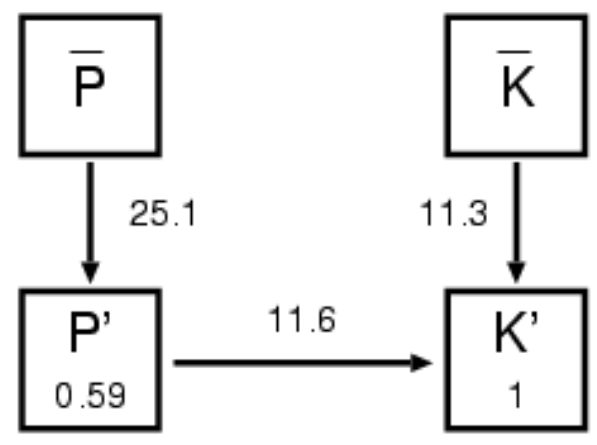

(c)

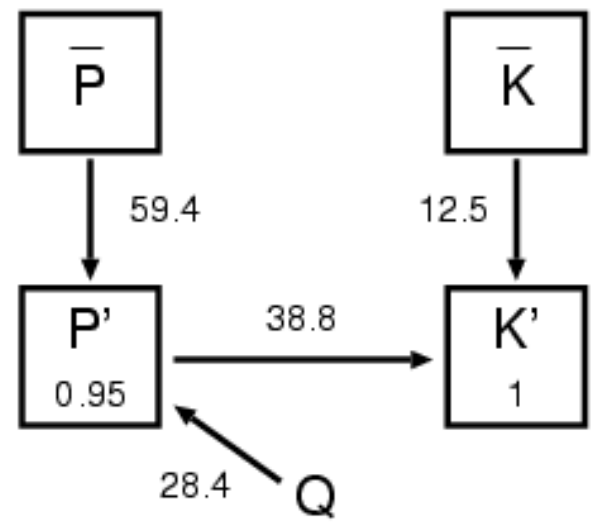

(b)

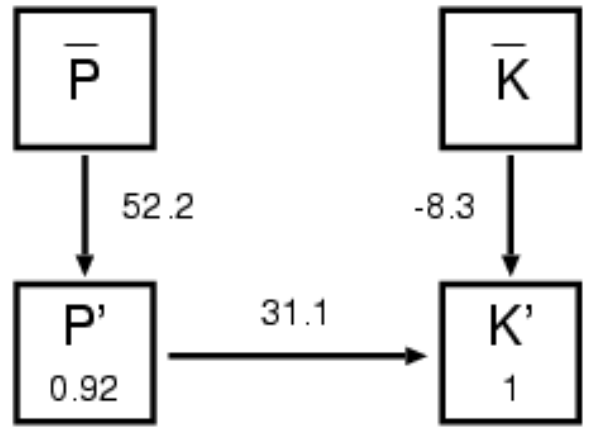

(d)

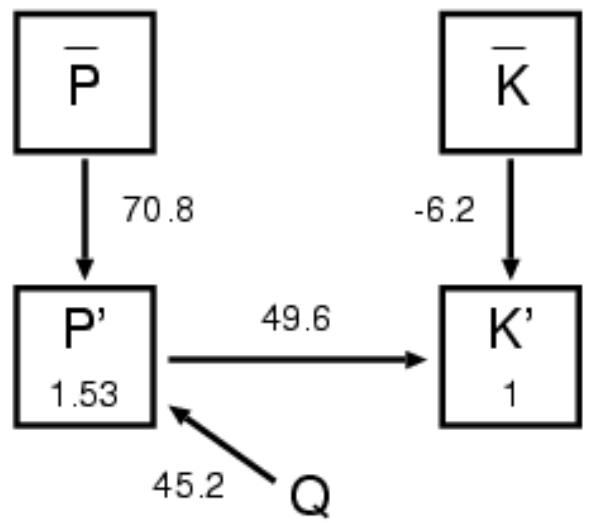

Fig. 5. As in Fig. 4b except for (a) DRY-POS, (b) DRY-NEG, (c) MOIST-POS and (d) MOIST-NEG waves.

present study. Near the bottom and top boundaries, however, the correlation shows different characteristics between the two waves: The DRY-POS wave has a low correlation because the phase line of $\theta$ significantly tilts eastward compared to that of $w$. This feature is not seen for DRY-NEG wave. For more quantitative discussion, Table 1 shows the difference of the phase between 4.54 and $0.46 \mathrm{~km}$ AGL for the several variables of each wave. A positive value for the phase difference means an eastward tilt with increasing height. Note that we do not use the values at the top and bottom boundaries to determine the phase because several variables vanish there. It is seen from Table 1 that, for DRY-POS wave, the tilt of $\theta$ (hereafter referred to as $\operatorname{TILT}(\theta)$ ) is 190 degrees and $\operatorname{TILT}(w)$ is 24 degrees. This large difference in the phase tilts (166 degrees) makes the correlation between $w$ and $\theta$ small, resulting in small $\left[P^{\prime}, K^{\prime}\right]$. For
DRY-NEG wave, on the other hand, the difference between $\operatorname{TILT}(\theta)$ and $\operatorname{TILT}(w)$ is relatively small (92 degrees), which results in the large correlation between $w$ and $\theta$, and therefore in larger $\left[P^{\prime}, K^{\prime}\right]$.

To understand the wave structures further, let us examine the horizontal pattern of the trough, ridge and streamlines at the lowest model level for DRY-POS and DRY-NEG waves in Fig. 8. The phase lines of troughs and ridges in DRY-POS wave align in the northwestsoutheast direction, whereas those in DRYNEG wave align in the northeast-southwest direction. Except for the directions of the phase lines, however, the patterns of both waves look quite similar: for DRY-POS (DRY-NEG) wave, the strong northwestward (northeastward) flow exists on the east side of the trough, while the strong southeastward (southwestward) flow is on the west side of the trough. The negative 
(a) $\mathrm{u}$

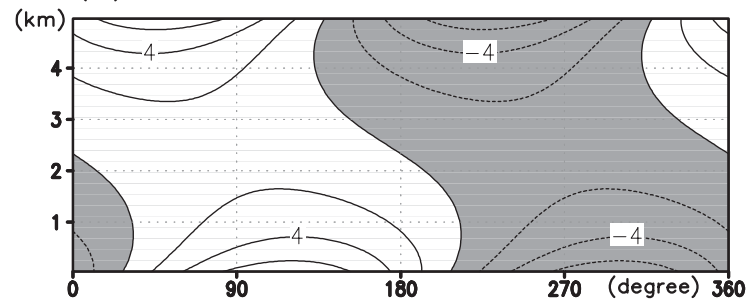

(c) $w$

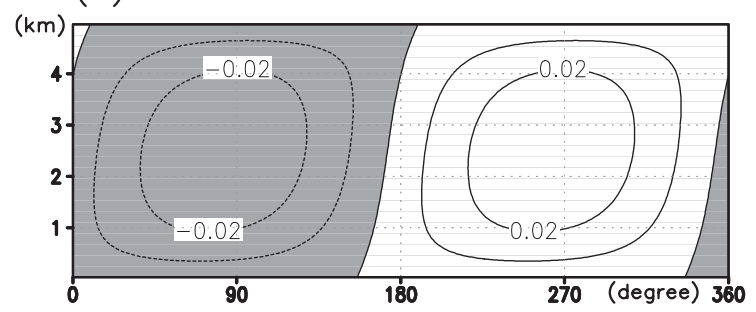

(e) $\theta$

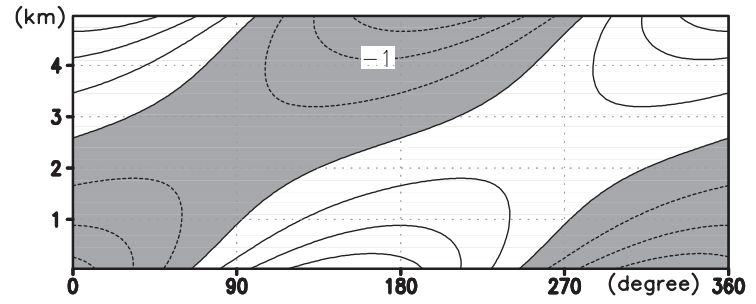

(g) zonal advection of $\theta$

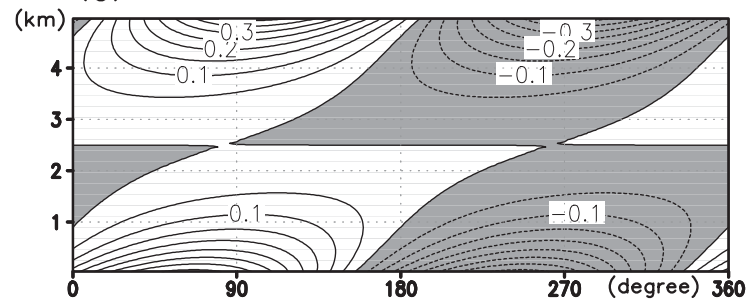

(i) vertical advection of $\theta$

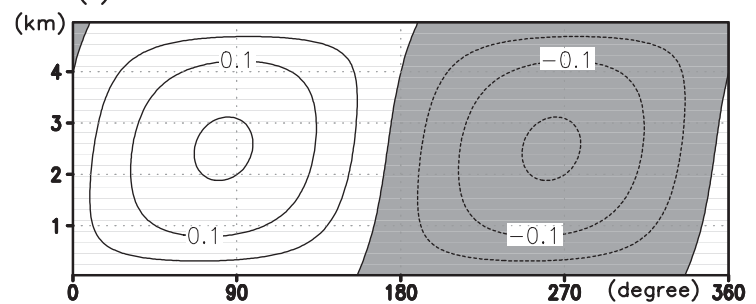

correlation between $u$ and $v$ in DRY-POS wave (Fig. 6) and the positive correlation in DRYNEG wave (Fig. 7) are closely related to the direction of the phase lines of the troughs and ridges in these waves. It is also noted that the streamlines near the troughs and ridges are (b) $\vee$

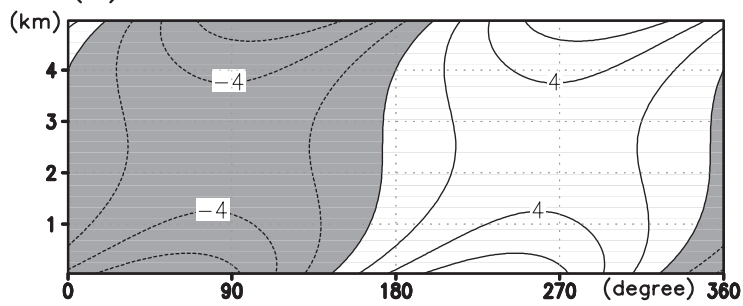

(d) $\phi$

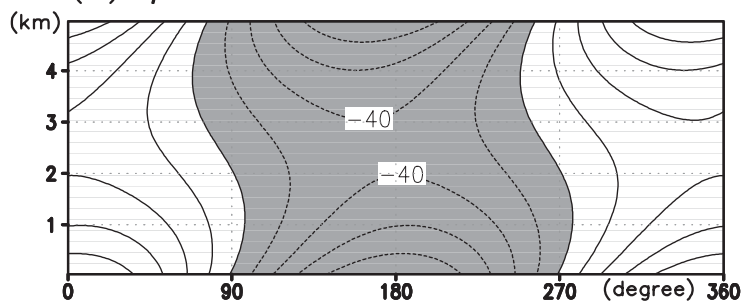

(f) tendency of $\theta$

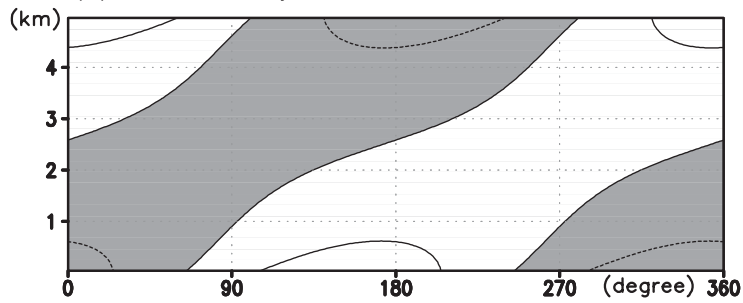

(h) meridional advection of $\theta$

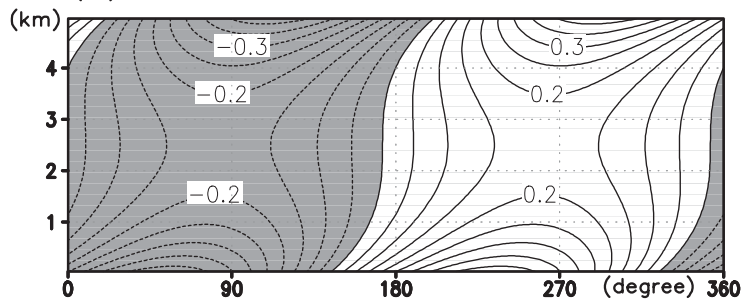

Fig. 6. As in Fig. 3 except for the DRY-POS wave. The contour intervals are (a), (b) $2 \mathrm{~ms}^{-1}$, (c) $0.01 \mathrm{~ms}^{-1}$, (d) $20 \mathrm{~m}^{2} \mathrm{~s}^{-2}$, (e) $0.5 \mathrm{~K}$, and (f)-(i) $0.05 \mathrm{Khr}^{-1}$.

not parallel to the isobars, demonstrating nongeostrophy.

\section{b. Moist waves}

We now show the results of the moist model. Figures 5c and 5d show the energy cycles for 
(a) $u$

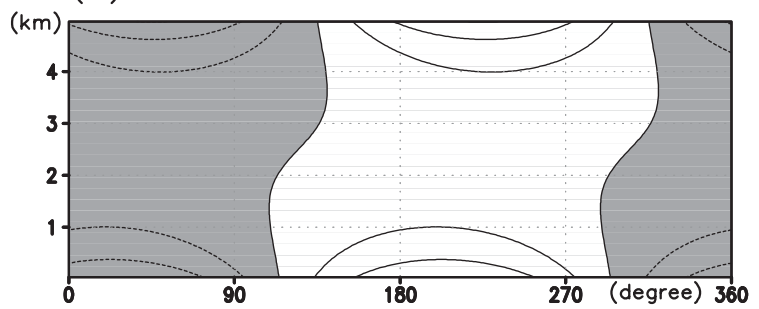

(c) $w$

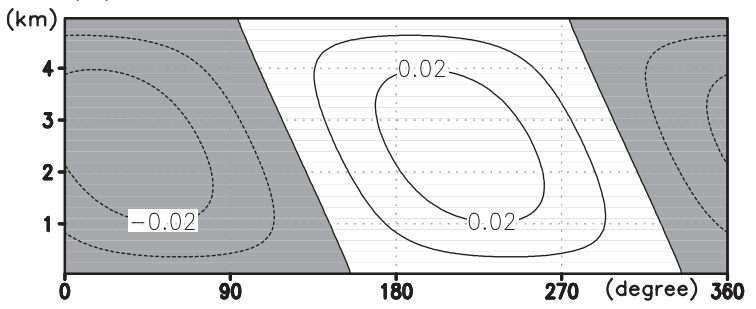

(e) $\theta$

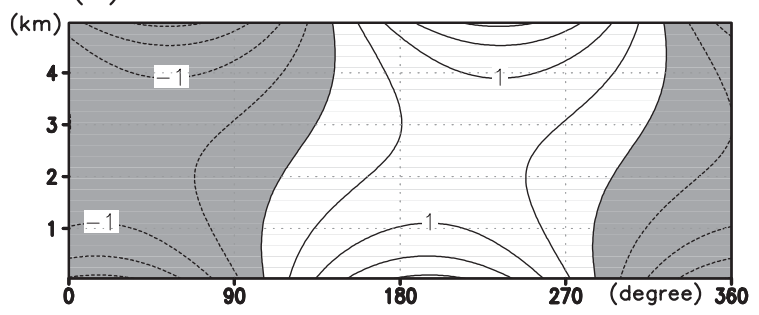

(g) zonal advection of $\theta$

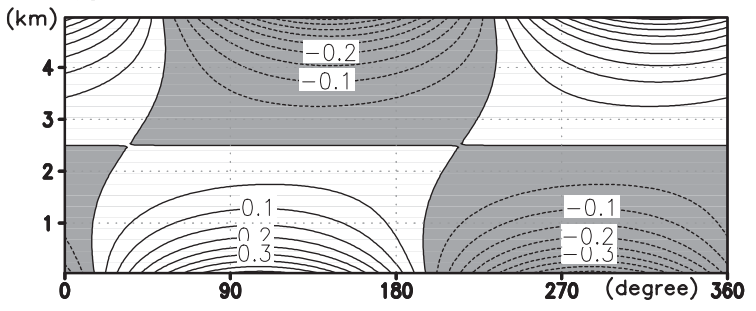

(i) vertical advection of $\theta$

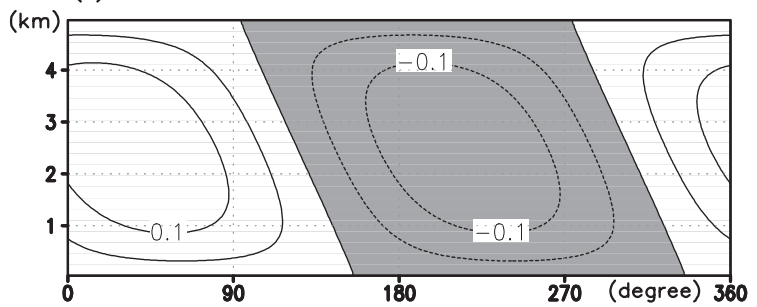

MOIST-POS and MOIST-NEG waves, respectively. As compared to the dry waves, $\left[P^{\prime}, K^{\prime}\right]$ is large in the moist waves. The increase of $\left[P^{\prime}, K^{\prime}\right]$ for the waves with positive meridional wavenumber $l$ (from 11.6 to $38.8 \times 10^{-6} \mathrm{~s}^{-1}$ ), (b) $v$

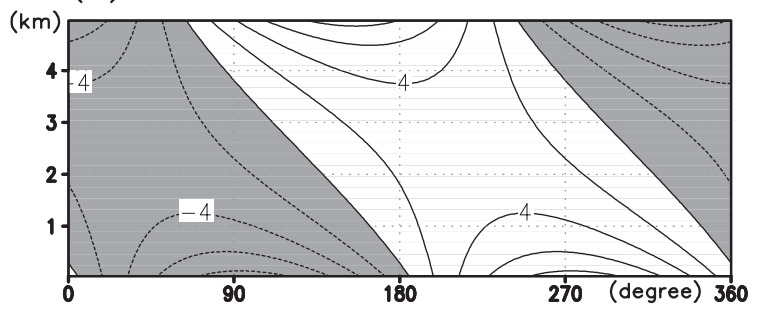

(d) $\phi$

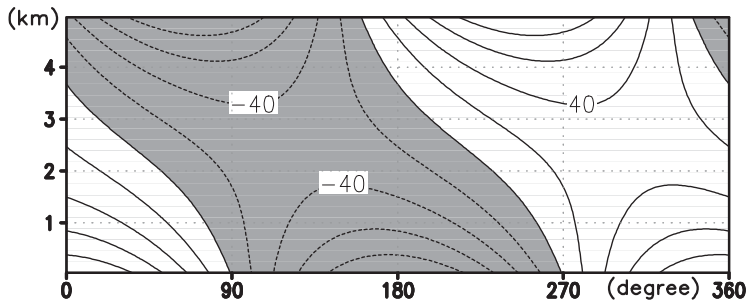

(f) tendency of $\theta$

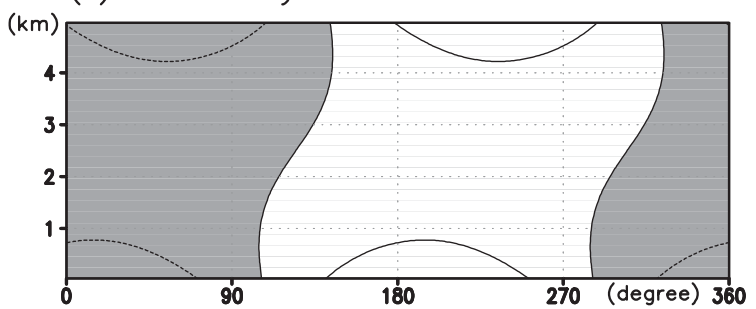

(h) meridional advection of $\theta$

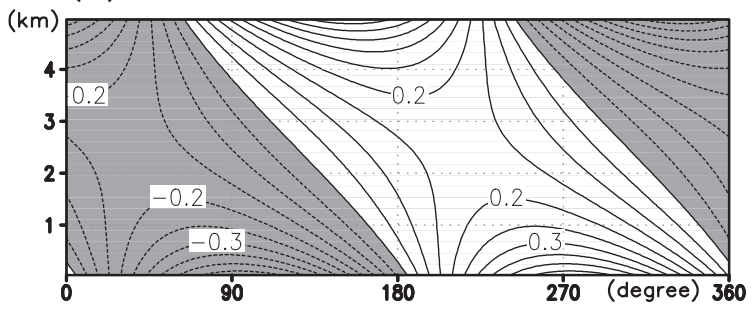

Fig. 7. As in Fig. 6 except for the DRYNEG wave.

however, is larger than that for the waves with negative $l$ (from 31.1 to $49.6 \times 10^{-6} \mathrm{~s}^{-1}$ ). The values of $\left[\bar{K}, K^{\prime}\right]$ for the moist waves, on the other hand, differ little from those for the dry waves. As a result, the ratio of $\left[\bar{K}, K^{\prime}\right]+\left[P^{\prime}, K^{\prime}\right]$ 
Table 1. The difference of the phase between 4.54 and $0.46 \mathrm{~km}$ AGL for the variables of the waves. The phase is given in the unit of degree and a positive value means an eastward tilt with increasing height. The variable $\theta_{\bar{u}}$ means zonal advection, $\theta_{v}$ meridional advection, $\theta_{w}$ vertical advection, and $\theta_{X}$ convective heating in the thermodynamic equation.

\begin{tabular}{|c|c|c|c|c|c|}
\hline Variable & DRY-POS & DRY-NEG & MOIST-POS & MOIST-NEG & $\begin{array}{c}\text { The most } \\
\text { unstable } \\
\text { wave } \\
\text { of the } \\
\text { T-mode }\end{array}$ \\
\hline$u$ & -79 & 25 & -109 & 11 & -21 \\
\hline$v$ & 33 & -103 & -3 & -127 & 132 \\
\hline$w$ & 24 & -52 & 10 & 54 & 73 \\
\hline$\phi$ & -22 & -98 & -85 & -142 & -50 \\
\hline$\theta$ & 190 & 40 & 121 & 9 & 218 \\
\hline$\theta_{v}$ & 33 & -103 & -3 & -127 & 132 \\
\hline$\theta_{v}+\theta_{w}$ & 35 & -115 & -6 & -143 & 190 \\
\hline$\theta_{v}+\theta_{w}+\theta_{\bar{u}}$ & 190 & 40 & 164 & 29 & 298 \\
\hline$\theta_{v}+\theta_{w}+\theta_{\bar{u}}+\theta_{X}$ & 190 & 40 & 121 & 9 & 218 \\
\hline
\end{tabular}

to $K^{\prime}$ for MOIST-POS wave $\left(51.3 \times 10^{-6} \mathrm{~s}^{-1}\right)$ is larger than that for MOIST-NEG wave $\left(43.4 \times 10^{-6} \mathrm{~s}^{-1}\right)$, which leads to the asymmetry of the growth rate with respect to the $k$ axis (see Fig. 2b). The increase of the ratio of $\left[\bar{P}, P^{\prime}\right]$ to $P^{\prime}$ from the dry model to the moist one is also larger for the waves with positive $l$ (from 25.1 to $59.4 \times 10^{-6} \mathrm{~s}^{-1}$ ) than for the negative $l$ (from 52.2 to $70.8 \times 10^{-6} \mathrm{~s}^{-1}$ ).
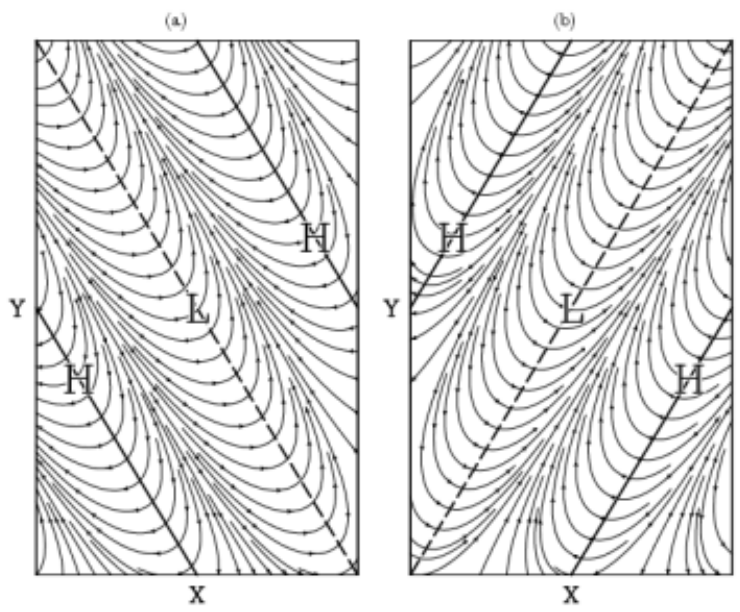

Fig. 8. Phase of the trough (indicated by the dashed line with "L"), ridge (indicated by the solid line with "H") and streamlines in the horizontal plane at the lowest model level (a) for the DRYPOS wave and (b) for the DRY-NEG wave.
Figures 9 and 10 show the zonal-vertical cross section of the several variables for MOIST-POS and MOIST-NEG waves, respectively. The most striking feature of MOISTPOS wave is that the difference between $\operatorname{TILT}(\theta)$ and $\operatorname{TILT}(w)$ is 111 degrees (see also Table 1) and is much smaller than that for DRY-POS wave. This results in a higher correlation between $w$ and $\theta$, and therefore in the larger increase of $\left[P^{\prime}, K^{\prime}\right]$ as seen in Fig. 5. The mechanism for the decrease of $\operatorname{TILT}(\theta)$ in MOIST-POS wave is to be discussed in the next section. Another notable feature is that the correlation between the meridional advection of $\theta$, and the convective heating in the upper layer, is better in MOIST-POS wave than in MOIST-NEG wave (compare Figs. 9h and 9j with Figs. $10 \mathrm{~h}$ and $10 \mathrm{j}$ ). This feature is also discussed in the next section.

\subsection{Moist symmetric instability mode and other modes}

For the moist model, we have noted that several new modes appear outside of the rectangle in Fig. 2b. These modes in general are difficult to distinguish clearly from each other. For small $k$ and large $|l|$, however, we find a mode that is clearly different from B-mode. If the growth rates of all unstable modes are plotted as a function of $k$ for $l=0.01 \mathrm{~km}^{-1}$, for example, we can confirm the curve that has a peak at $k=0$, and is apparently different from B-mode which dominates for $k>0.02 \mathrm{~km}^{-1}$. 
(a) $u$

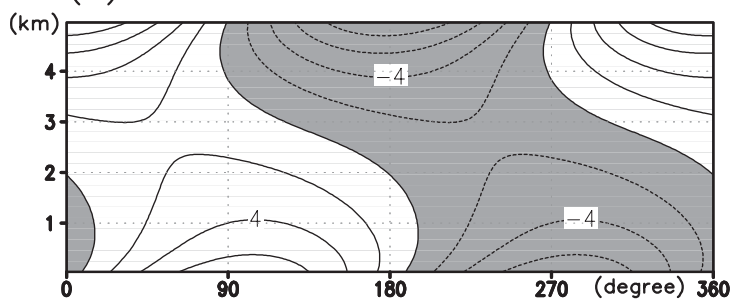

(c) $w$

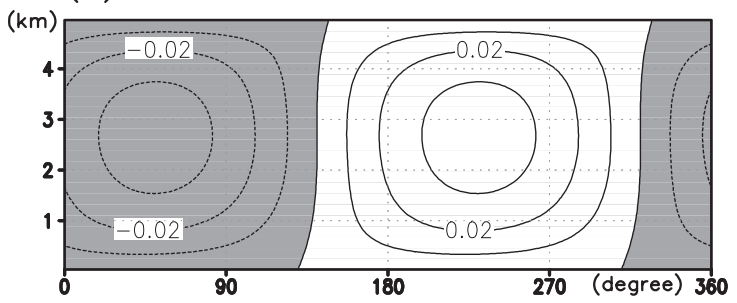

(e) $\theta$

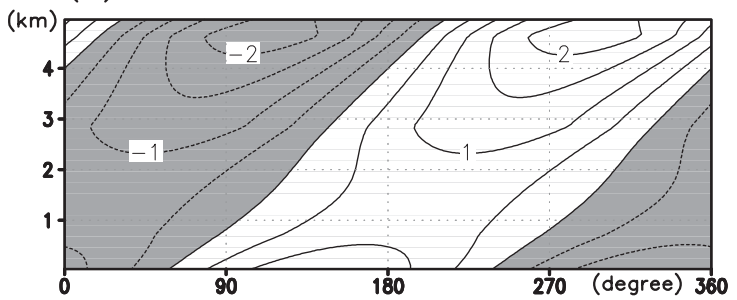

(g) zonal advection of $\theta$

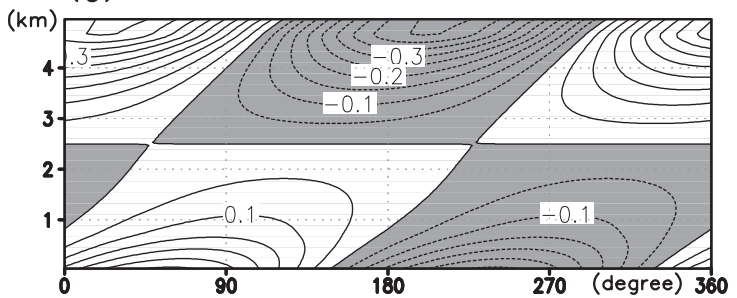

(i) vertical advection of $\theta$

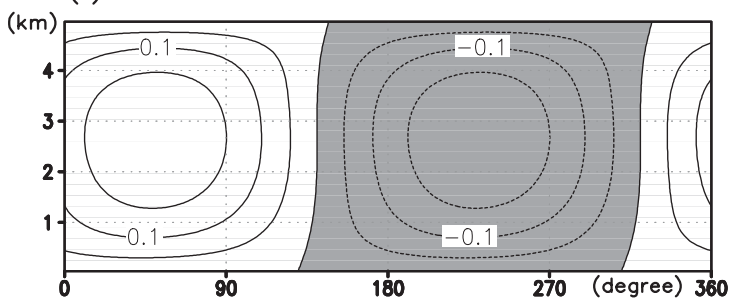

(b) $v$

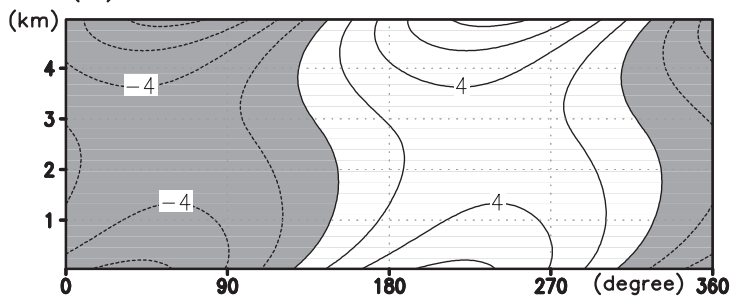

(d) $\phi$

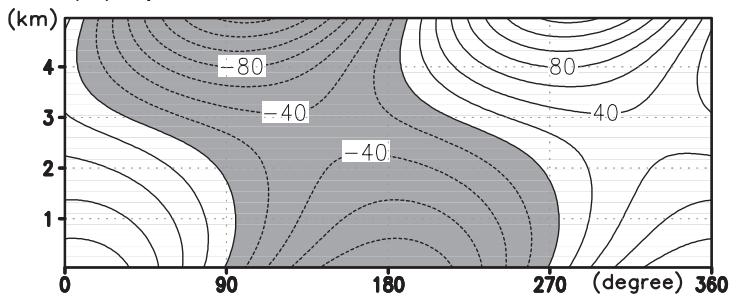

(f) tendency of $\theta$

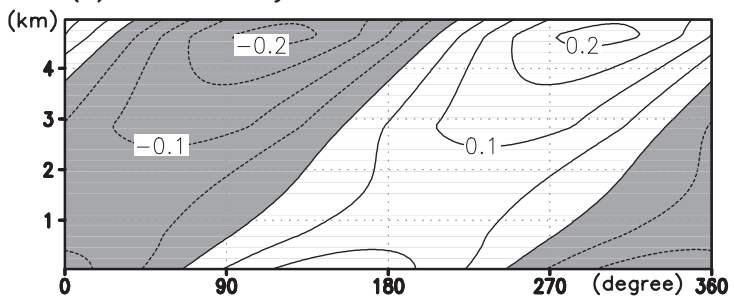

(h) meridional advection of $\theta$

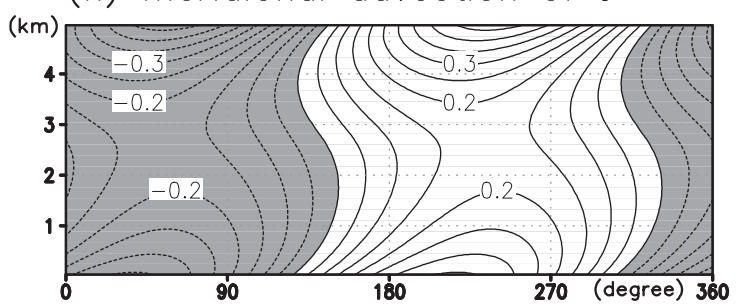

(j) convective heating

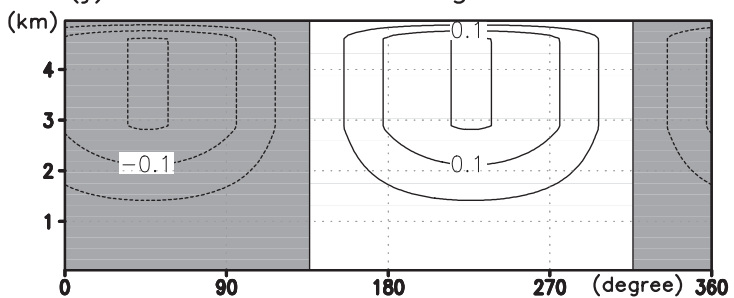

Fig. 9. As in Fig. 6 except for the MOIST-POS wave.

This mode is hereafter referred to as "S-mode". Here, we will briefly examine the structure of the S-mode with $k=0$ and $l=0.012 \mathrm{~km}^{-1}$ (denoted by the cross in Fig. 2b). Figure 11 shows the meridional-vertical cross section of the sev- eral variables for S-mode wave. Note that the wave has no zonal structure because the zonal wavenumber is zero. All the perturbations except for $u$ and for $\theta$ below $1 \mathrm{~km}$ AGL exhibit a northward tilt with height. Several features 
(a) $u$

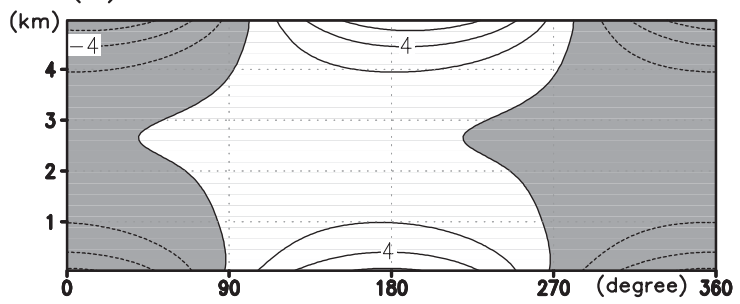

(c) $w$

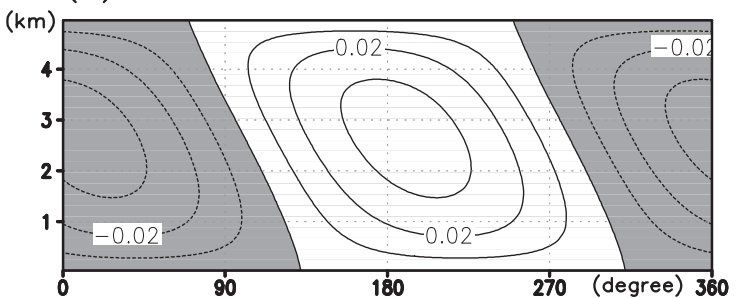

(e) $\theta$

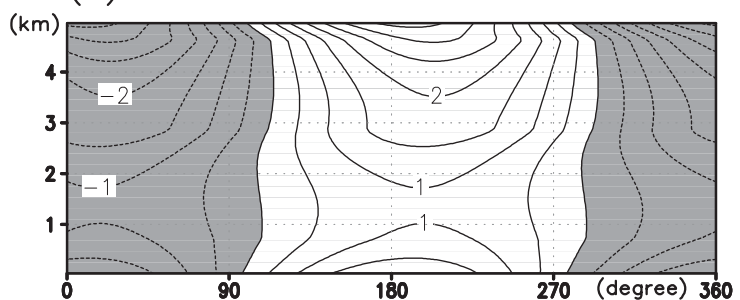

(g) zonal advection of $\theta$

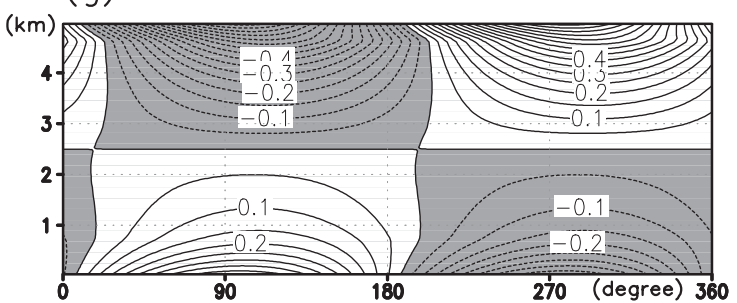

(i) vertical advection of $\theta$

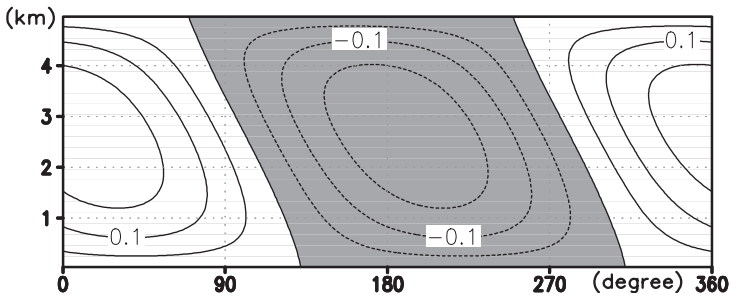

(b) $v$

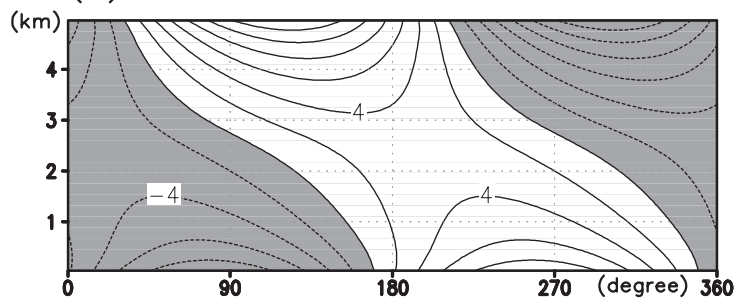

(d) $\phi$

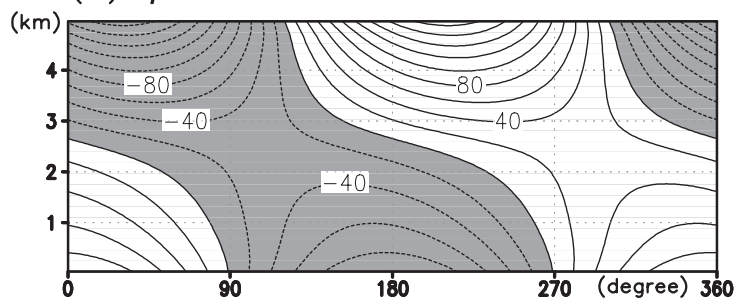

(f) tendency of $\theta$

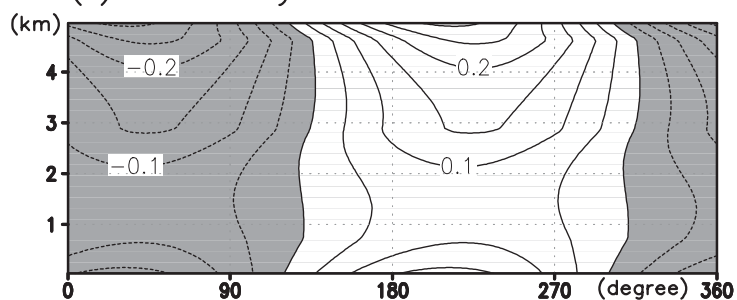

(h) meridional advection of $\theta$

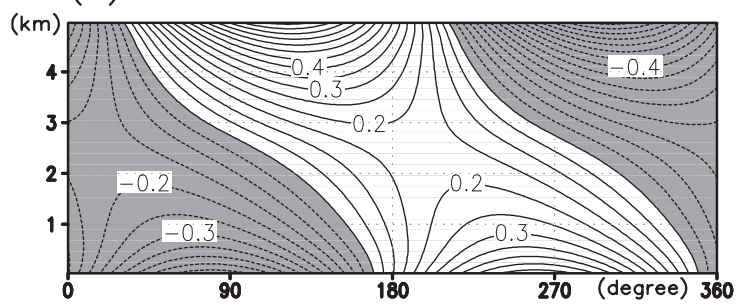

(j) convective heating

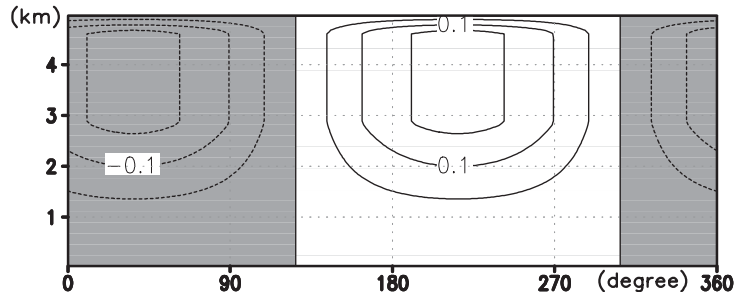

Fig. 10. As in Fig. 6 except for the MOIST-NEG wave.

resemble those of dry symmetric instability shown in Fig. 10c of Tokioka (1970) (Note that although he showed only the zonal-vertical cross section, it has the same pattern as meridional-vertical cross section in the case that both $k$ and $l$ are positive): the correlation between $w$ and $\phi$ is negative near the bottom boundary, while it is positive near the top boundary; the correlation between $w$ and $\theta$ is positive at the mid-level. Note that this mode 
(a) $u$

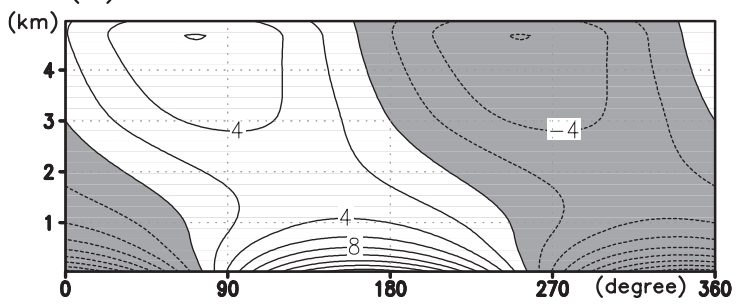

(c) $w$

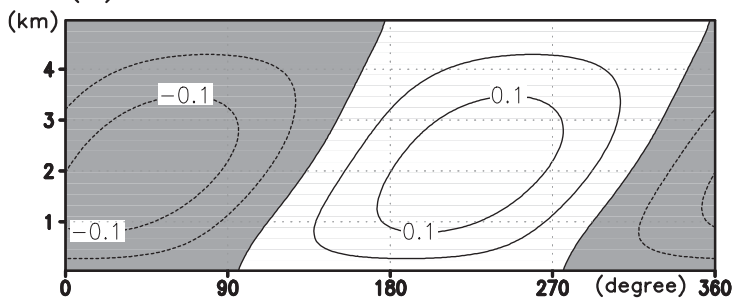

(e) $\theta$

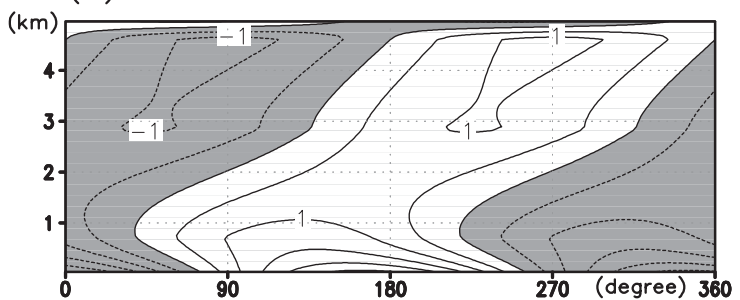

(b) $v$

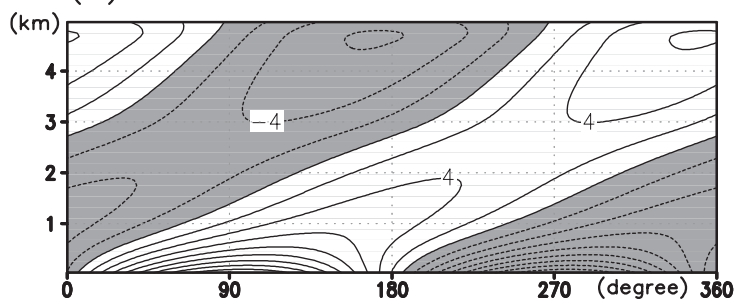

(d) $\phi$

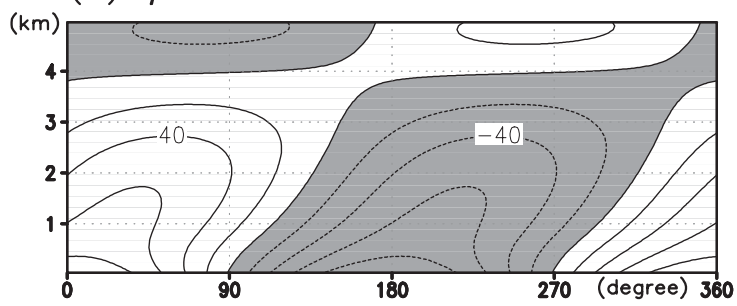

(f) convective heating

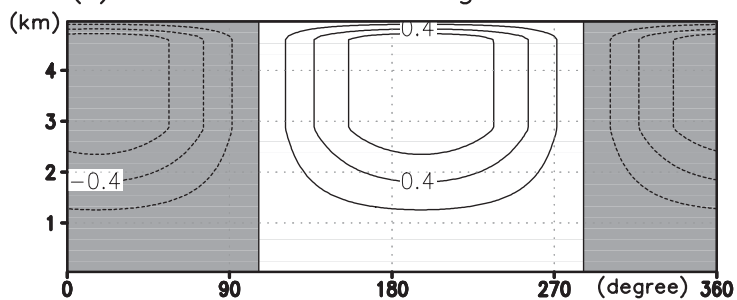

Fig. 11. Meridional-vertical cross section of the S-mode wave $\left(k=0\right.$ and $\left.l=0.012 \mathrm{~km}^{-1}\right)$. (a) $u$ (c.i. $\left.=2 \mathrm{~ms}^{-1}\right) ; \quad\left(\right.$ b) $v \quad\left(\right.$ c.i. $\left.=2 \mathrm{~ms}^{-1}\right) ; \quad$ (c) $w \quad\left(\right.$ c.i. $\left.=0.05 \mathrm{~ms}^{-1}\right) ; \quad$ (d) $\phi \quad\left(\right.$ c.i. $\left.=20 \mathrm{~m}^{2} \mathrm{~s}^{-2}\right) ; \quad$ (e) $\theta$ $($ c.i. $=0.5 \mathrm{~K})$; (f) convective heating $\left(\right.$ c.i. $\left.=0.2 \mathrm{Khr}^{-1}\right)$. The shaded regions indicate the negative values.

does not appear in the dry model (see Fig. 2a) when $R i$ is larger than unity (Stone 1966). Therefore, S-mode seems to be a kind of symmetric instability wave that is destabilized by the convective heating.

The energy cycle of S-mode has also notable features. The ratio of $P^{\prime}$ to $K^{\prime}$ is 0.35 . The conversion $\left[Q, P^{\prime}\right]$ is $47.4 \times 10^{-6} \mathrm{~s}^{-1}$ and is larger than $\left[\bar{P}, P^{\prime}\right]\left(33.0 \times 10^{-6} \mathrm{~s}^{-1}\right)$. The conversion $\left[P^{\prime}, K^{\prime}\right]$ is $57.1 \times 10^{-6} \mathrm{~s}^{-1}$ and is considerably larger than $\left[\bar{K}, K^{\prime}\right]\left(9.9 \times 10^{-6} \mathrm{~s}^{-1}\right)$. The feature that $\left[P^{\prime}, K^{\prime}\right]$ is larger than $\left[\bar{K}, K^{\prime}\right]$ is also similar to that of the dry symmetric instability (e.g., Tokioka 1970). The asymmetry of the energy cycle with respect to the $k$-axis is relatively small for S-mode with $k=0$ (not shown).

We have also found another mode with $k$ larger than that shown in Fig. 2b (e.g., $k=$ $0.05 \mathrm{~km}^{-1}$ and $l=0 \mathrm{~km}^{-1}$; not shown). This mode shows two peaks of geopotential amplitude at the top and bottom boundaries, and has a rapid change of geopotential phase around $4.5 \mathrm{~km}$ AGL. Using the quasi-geostrophic model and a top-hat heating profile, Mak (1994) found two additional modes that are different from the ordinary baroclinic wave: an upper mode and a lower mode. The upper (lower) mode has two peaks of streamfunction at the top (bottom) boundary and the level above (below) which the heating vanishes. $\mathrm{He}$ interpreted these modes in terms of wave resonant interactions from a viewpoint of potential vorticity. In order to examine if Mak's interpretation is applicable to our mode, we calculated the stability for a top-hat heating profile. It turned out that our mode is highly nongeostrophic and it is not easy to discuss the relationship between our mode and Mak's. It should also be noted that our mode seems to be 
different from the computational mode for Lorenz grid model (Arakawa and Moorthi 1988) since the amplitude of streamfunction of their mode is trapped at only one boundary.

\section{Discussion}

\subsection{Asymmetry of dry waves with respect to the meridional wavenumber}

In this subsection, we examine why the structure and energy cycle of the dry wave have an asymmetry with respect to the sign of the meridional wavenumber although its growth rate is symmetric. For DRY-POS wave, the correlation between $u$ and $w$ is negative, which leads to a positive value of $\left[\bar{K}, K^{\prime}\right]$. For DRYNEG wave, on the other hand, the correlation is positive, giving a negative value of $\left[\bar{K}, K^{\prime}\right]$. This can be explained as follows: For a sloped convection like a baroclinic wave, warm (cold) air moves northward (southward) and upward (downward). Thus, both waves have a positive correlation between $v$ and $w$ as seen in Figs. 6 and 7. The correlation between $u$ and $v$, on the other hand, is negative in DRY-POS wave, and positive in DRY-NEG wave, which is simply determined by the phase line of the troughs and ridges (see Fig. 8). As a result, the correlation between $u$ and $w$ becomes negative for DRY-POS wave and positive for DRY-NEG wave.

The difference of $\left[\bar{K}, K^{\prime}\right]$ between DRY-POS and DRY-NEG waves is exactly compensated by the difference of $\left[P^{\prime}, K^{\prime}\right]$ : the conversion $\left[P^{\prime}, K^{\prime}\right]$ is larger for DRY-NEG wave than for DRY-POS wave. Thus, the two waves have the same growth rate. The difference of $\left[P^{\prime}, K^{\prime}\right]$ for the two waves might be explained as follows: As was seen in Fig. 6, the axis of $\theta$ field has a remarkable eastward tilt for DRY-POS wave, resulting in the low correlation between $w$ and $\theta$. In order to understand the structure of $\theta$ field, the contribution of the terms in the thermodynamic equation to the tilt of $\theta$ field has been examined (Table 1): The variables $\theta_{\bar{u}}, \theta_{v}, \theta_{w}$ and $\theta_{X}$ in Table 1 denotes the zonal advection $-i k \bar{u} \hat{\theta}$, meridional advection $f \Lambda \hat{v} / g \alpha$, vertical advection $-S \hat{w}$ and convective heating $X_{\theta}$, respectively. The TILT $\left(\theta_{v}+\theta_{w}\right)$, for example, gives the tilt of $\theta$ field resulting from the meridional advection plus vertical advection. Firstly, $\quad\left[\operatorname{TILT}\left(\theta_{v}\right)-\operatorname{TILT}(\phi)\right]$ is remarkably larger for DRY-POS wave (55 degrees) than for
DRY-NEG wave ( -5 degrees). Secondly, if the vertical advection is added, $\operatorname{TILT}\left(\theta_{v}+\theta_{w}\right)$ is little different from $\operatorname{TILT}\left(\theta_{v}\right)$ for both waves. Thirdly, the addition of the zonal advection causes a large increase in $\operatorname{TILT}\left(\theta_{v}+\theta_{w}+\theta_{\bar{u}}\right)$. The increase of the tilt, however, is of the same magnitude for both DRY-POS and DRY-NEG waves; i.e., $\operatorname{TILT}\left(\theta_{v}+\theta_{w}+\theta_{\bar{u}}\right)-\operatorname{TILT}\left(\theta_{v}+\theta_{w}\right)$ is 155 degrees for both waves. Thus, the above analysis shows that the critical mechanism which causes the significantly large $[\operatorname{TILT}(\theta)-$ $\operatorname{TILT}(\phi)]$ for DRY-POS wave is the meridional advection.

The large difference of $\left[\operatorname{TILT}\left(\theta_{v}\right)-\operatorname{TILT}(\phi)\right]$ between DRY-POS and DRY-NEG waves might be explained as follows: At the lowest level, as is seen in Fig. 8, the distance between the location of the trough and the maximum southerly wind to the east of the trough for DRY-POS wave is smaller than that for DRY-NEG wave. At the uppermost level (not shown), on the other hand, the distance is larger for DRYPOS wave than for DRY-NEG wave. As a result, [TILT $\left(\theta_{v}\right)-\operatorname{TILT}(\phi)$ ] for DRY-POS wave is larger than that for DRY-NEG wave, which, in turn, results in the significantly large $[\operatorname{TILT}(\theta)-\operatorname{TILT}(\phi)]$ for DRY-POS wave. Note that $[\operatorname{TILT}(w)-\operatorname{TILT}(\phi)]$ for both waves are nearly similar (see $w$ and $\phi$ in Table 1). Therefore, for DRY-POS wave, the correlation between $w$ and $\theta$ is weak and $\left[P^{\prime}, K^{\prime}\right]$ is small compared to DRY-NEG wave.

\subsection{Asymmetry of moist waves with respect to the meridional wavenumber}

For the moist waves, we have seen that not only their structures but also their growth rates have a remarkable asymmetry with respect to the sign of the meridional wavenumber (Figs. 2b, 9 and 10). In this subsection, we attempt to understand what causes this asymmetry. Compared to DRY-POS wave, MOISTPOS wave has a large correlation between $w$ and $\theta$ (see Figs. 6c, 6e, 9c and 9e). As a result, the difference of $\left[P^{\prime}, K^{\prime}\right]$ between MOIST-POS and MOIST-NEG waves $(38.8$ and $49.6 \times$ $10^{-6} \mathrm{~s}^{-1}$, respectively; see Figs. $5 \mathrm{c}$ and $5 \mathrm{~d}$ ) is smaller than that between DRY-POS and DRY-NEG waves $\left(11.6\right.$ and $31.1 \times 10^{-6} \mathrm{~s}^{-1}$, respectively; see Figs. 5a and 5b). As was mentioned in Subsection 3.3, the large correlation between $w$ and $\theta$ in MOIST-POS wave 
results from the small tilt of $\theta$ field compared to DRY-POS wave.

The mechanism by which $\operatorname{TILT}(\theta)$ is reduced for MOIST-POS wave is diagnosed by examining the four terms in the thermodynamic equation: the zonal advection, the meridional advection, the vertical advection and the convective heating. Table 1 shows that $\operatorname{TILT}\left(\theta_{v}+\theta_{w}\right)$ relative to $\operatorname{TILT}(\phi)$ is larger for MOIST-POS wave (79 degrees) than MOISTNEG wave ( -1 degrees). For both waves, the zonal advection further increase the eastward tilt (see " $\theta_{v}+\theta_{w}$ " and " $\left.\theta_{v}+\theta_{w}+\theta_{\bar{u}} "\right)$. The mechanism mentioned above also operates for the dry waves as seen in the previous section. For the moist model, the convective heating, in addition, has an important role to determine $\operatorname{TILT}(\theta)$. For MOIST-POS wave, the maximum convective heating (Fig. 9j) occurs on the west side of the maximum potential temperature tendency (Fig. 9f) in the upper layer. This means the convective heating counteracts the zonal advection to cause the large $\operatorname{TILT}(\theta)$ (compare " $\theta_{v}+\theta_{w}+\theta_{\bar{u}}$ " with " $\theta_{v}+\theta_{w}+\theta_{\bar{u}}+\theta_{X}$ " in Table 1). For MOIST-NEG wave, on the other hand, the maximum convective heating (Fig. 10j) occurs nearly at the same location of the maximum potential temperature tendency (Fig. 10f) in the upper layer and has little effect on $\operatorname{TILT}(\theta)$. Thus, the convective heating for MOIST-POS wave is more effective in reducing $\operatorname{TILT}(\theta)$ and thus contributing to increase the correlation between $w$ and $\theta$ or $\left[P^{\prime}, K^{\prime}\right]$.

\subsection{The effect of convective heating on baroclinic instability}

In the present model, the proper location of convective heating in the upper layer that increases the correlation between $w$ and $\theta$ leads to the large value of $\left[P^{\prime}, K^{\prime}\right]$ for MOIST-POS wave. There have been a number of studies on the effects of convective heating on baroclinic instability: Moorthi and Arakawa (1985) studied a stability of a baroclinic flow using a different parameterization in which the convective heating depends on the vertical velocity at all levels. They demonstrated that their parameterization results in larger growth rates than those for the CISK-type parameterization used in the present study. They also noted that their parameterization makes the mechanism somewhat similar to that with a reduced static stability. Sardie and Warner (1983) also obtained a similar result for a condensational heating which depends on the vertical velocity at each level. In terms of the energy cycle, the condensational heating which depends on the vertical velocity at each level tends to make the correlation between $w$ and $\theta$ larger, and to increase $\left[P^{\prime}, K^{\prime}\right]$. The CISK-type convective heating, on the other hand, does not necessarily occurs within the updraft region in the upper layer, and therefore may not contribute to increase $\left[P^{\prime}, K^{\prime}\right]$. In fact, MOIST-NEG wave in the present study has a weaker correlation between the vertical velocity and the convective heating than MOIST-POS wave does (see Figs. 9 and 10).

Moorthi and Arakawa (1985) also demonstrated that, when the convective heating occurs at relatively high levels, the horizontal divergence in the upper part of the updraft region does not interact with the baroclinic layer in the lower troposphere, resulting in a less effective amplification of the vortex. In the present case, however, the level of the horizontal divergence is confined to the baroclinic layer and a strong interaction is assured.

It is certain that the interaction between the convective heating and the baroclinic instability depends on the atmospheric environment, the upper boundary condition, and convective heating parameterization. The present study has shown, however, that not only the amount of convective heating, but also its location are important for determining how much energy is extracted from baroclinicity and thus affecting the growth rate.

\section{Summary and concluding remarks}

Non-geostrophic non-hydrostatic baroclinic instability with and without convective heating is re-examined with an emphasis on the structures of the growing modes and their energy cycles for the case of a moderate Richardson number $(R i=2)$.

When the convective heating is absent, only the baroclinic instability mode has a positive growth rate. The growth rate is maximum when the meridional wave number $l$ vanishes, and is symmetric with respect to $l=0$; i.e., the growth rates for $\pm l$ are equal. The energy budget for both waves, however, show a marked difference: For the wave with $l>0$, both $\left[\bar{K}, K^{\prime}\right]$ 
and $\left[P^{\prime}, K^{\prime}\right]$ operate with similar magnitude to increase $K^{\prime}$. For the wave with $l<0$, on the other hand, $\left[P^{\prime}, K^{\prime}\right]$ is larger than that for the wave with $l>0$, and $\left[\bar{K}, K^{\prime}\right]$ is negative. The sum of $\left[\bar{K}, K^{\prime}\right]$ and $\left[P^{\prime}, K^{\prime}\right]$ for both waves, however, are equal.

When the convective heating is included, the structures, energy budgets and growth rates of the baroclinic instability mode (B-mode) is modified, and several additional modes, which were not studied by Tokioka (1973), are destabilized. The results with the convective heating may be summarized as follows:

1. The fastest growing B-mode has an eastward tilt of the trough with increasing height in the lower layer. As expected from the hydrostatic balance, a cold air exists to the east of the surface trough. The thermodynamic diagnosis reveals that this cold air is caused due to an updraft driven by the convective heating at the upper-levels;

2 . The increase of the growth rate for B-mode by the inclusion of the convective heating is larger for the wave with $l>0$ than for that with $l<0$. Energy budget analysis shows the convective heating hardly affects $\left[\bar{K}, K^{\prime}\right]$, but increases $\left[P^{\prime}, K^{\prime}\right]$ more effectively for the wave with $l>0$ than for that with $l<0$. For the wave with $l>0$, the baroclinic wave without convective heating has a weaker correlation between $w$ and $\theta$ than that for the wave with $l<0$. Thus, the convective heating which tends to increase the correlation between $w$ and $\theta$ contributes more effectively to increase $\left[P^{\prime}, K^{\prime}\right]$; and,

3 . For small zonal wavenumber and large positive and negative meridional wavenumbers, the convective heating destabilizes a new mode (S-mode) which has characteristics similar to those of symmetric instability in the dry atmosphere. Note that this mode does not appear in the dry experiment for $R i>1$ (Stone 1966).

When one would like to apply the present results to meso-scale disturbances in the real atmosphere, one needs to care about the following problems: first, for the disturbances on the Baiu front, the basic field that assumes meridional homogeneity of zonal flow and potential temperature gradient may be oversimplified; second, it has been suggested that only the
CISK mechanism seems to be dominant for some types of polar lows (Rasmussen 1979; Rasmussen 1985), while the baroclinic instability with convective heating seems to play an important role for other types (Sardie and Warner 1983; Craig and Cho 1988); the present analysis is only valid for the latter types; and, third, the parameterization that an "evaporative cooling" occurs in downdraft regions in a manner opposite to the convective heating is highly unrealistic. Nevertheless, the present results give a good theoretical basis to understand the meso-scale disturbances in which both baroclinicity and convective heating play an important role. We are currently performing a cloud-resolving numerical study of baroclinic waves in a moist atmosphere. Its results and a comparative discussion with the present theoretical model will be reported elsewhere.

\section{Acknowledgements}

This work was partly supported by CREST of JST (Japan Science and Technology Corporation) "Studies on Structure and Formation/ Development Mechanisms of Mesoscale Convective Systems", Grant-in-Aid for Scientific Research (A) No. 14340138 of Japan Society for the Promotion of Science (JSPS), and a Grant for the 21st Century COE Program "Predictability of the Evolution and Variation of the Multi-scale Earth System: An Integrated COE for Observational and Computational Earth Science" from the Ministry of Education, Culture, Sports, Science, and Technology of Japan. W.Y. would like to thank Dr. Tatsushi Tokioka of Meteorological College for kindly providing precious information on his paper (Tokioka 1973). We appreciate useful comments from Prof. Mankin Mak and one anonymous reviewer.

\section{Appendix}

In this Appendix, we will first clarify the relationship between the non-geostrophic nonhydrostatic system (present study, T73 and Gambo 1976) and the quasi-geostrophics system (Mak 1982; Sardie and Warner 1983 and Moorthi and Arakawa 1985) for the dry experiment. Second, we will prove that the growth rate for the dry experiment is an even function of the wavenumber, even though its structure is asymmetric with respect to $l=0$. 
To this end, it is convenient to use a nondimensionalized equation system, where dimensional quantities (with prime) are scaled as follows:

$$
\begin{aligned}
\left(x^{\prime}, y^{\prime}\right) & =L(x, y), \\
\left(k^{\prime}, l^{\prime}\right) & =\frac{1}{L}(k, l), \\
z^{\prime} & =H z, \\
t^{\prime} & =T t, \\
\sigma^{\prime} & =\frac{1}{T} \sigma, \\
w^{\prime} & =W w, \\
\left(u^{\prime}, v^{\prime}\right) & =U(u, v), \\
\phi^{\prime} & =\Phi \phi, \\
\theta^{\prime} & =\Theta \theta, \\
\bar{u}^{\prime} & =\bar{U} \bar{u},
\end{aligned}
$$

where $L=\frac{N H}{f}$ is the Rossby radius of deformation, $N(\equiv \sqrt{g \alpha S})$ the Brunt-Väisäla frequency, $H=z_{0}, \quad \bar{U}=\Lambda H, \quad T=\frac{L}{\bar{U}}=\frac{N}{f \Lambda}, \quad U=\frac{N}{f} W, \quad \Phi=$ $\frac{N^{2} W H}{f}$ and $\Theta=\frac{N^{2} W}{g \alpha f}$.

We also define the following non-dimensional parameters:

$$
\begin{array}{lrl}
\text { Aspect ratio: } & \delta & \equiv \frac{L}{H}=\frac{N}{f}, \\
\text { Rossby number: } & \varepsilon & \equiv \frac{\bar{U}}{f L}=\frac{\Lambda}{N}, \quad \text { and } \\
\text { Richardson number: } & R i & \equiv \frac{N^{2}}{\Lambda^{2}}\left(=\varepsilon^{-2}\right) .
\end{array}
$$

Then the non-dimensional form of Eqs. (3)-(7) for the dry experiment may be written as,

$$
\begin{aligned}
& i \varepsilon(k \bar{u}-\sigma) u+\varepsilon w-v=-i k \phi, \\
& i \varepsilon(k \bar{u}-\sigma) v+u=-i l \phi, \\
& i \varepsilon \delta^{-2}(k \bar{u}-\sigma) w-\theta=-d \phi / d z, \\
& i \varepsilon(k \bar{u}-\sigma) \theta-\varepsilon v+w=0, \\
& i k u+i l v+d w / d z=0 .
\end{aligned}
$$

And the equations for the horizontallyaveraged eddy kinetic energy and eddy available potential energy may be written as,

$$
\begin{aligned}
& \varepsilon \frac{\partial}{\partial t}\left(\frac{u^{2}+v^{2}+\delta^{-2} w^{2}}{2}\right)=\varepsilon \overline{u w}+\overline{w \theta}-\frac{d \overline{w \phi}}{d z} \\
& \varepsilon \frac{\partial}{\partial t}\left(\frac{\theta^{2}}{2}\right)=\varepsilon \overline{v \theta}-\overline{w \theta}
\end{aligned}
$$

Note that, in a quasi-geostrophic system, $\varepsilon \ll 1$ is assumed and $w$ is $O(\varepsilon)$. Therefore, the term $\varepsilon \overline{u w}$ is $O\left(\varepsilon^{2}\right)$ and is neglected.

Elimination of $u, v, \phi$ and $\theta$ from the Eqs. (A-1)-(A-5) by the similar process to Tokioka (1970; see Eqs. (2-9)-(2-20)) leads to the following equation for $w$ :

$$
\begin{aligned}
\varepsilon(k \bar{u} & -\sigma)\left\{\varepsilon^{2}(k \bar{u}-\sigma)^{2}-1\right\} \frac{d^{2} w}{d z^{2}} \\
& +2 \varepsilon\{k-i l \varepsilon(k \bar{u}-\sigma)\} \frac{d w}{d z} \\
& +\left\{\left(k^{2}+l^{2}\right) \varepsilon(k \bar{u}-\sigma)+2 i \varepsilon^{2} k l\right. \\
& \left.-\delta^{-2}\left(k^{2}+l^{2}\right) \varepsilon^{3}(k \bar{u}-\sigma)^{3}\right\} w=0 .
\end{aligned}
$$

The boundary conditions are $w=0$ at $z=0$ and 1. Introducing a new independent variable $X\left(\equiv k \bar{u}-\sigma=k\left(z-\frac{1}{2}\right)-\sigma\right)$, we have

$$
\begin{gathered}
\varepsilon X\left\{(\varepsilon X)^{2}-1\right\} \frac{d^{2} w}{d X^{2}}+2 \varepsilon\left(1-i \frac{l}{k} \varepsilon X\right) \frac{d w}{d X} \\
+\left\{\left(1+\frac{l^{2}}{k^{2}}\right) \varepsilon X+2 i \varepsilon^{2} \frac{l}{k}\right. \\
\left.-\delta^{-2}\left(1+\frac{l^{2}}{k^{2}}\right)(\varepsilon X)^{3}\right\} w=0 .
\end{gathered}
$$

The boundary conditions are $w=0$ at $X=$ $-\sigma-\frac{k}{2}$ and $-\sigma+\frac{k}{2}$. Equation (A-9) corresponds to Eq. (2-20)" of Tokioka (1970). When hydrostatic approximation $(\delta \gg 1)$ is assumed, we have the similar equation to Eq. (II-11) of Eady (1949) and to Eq. (2-11) with $Q=0$ of Gambo (1976). Eady (1949) simplified the equation further by assuming a small Rossby number and a large Richardson number $(\varepsilon \ll 1)$.

When $\varepsilon$ is not neglected, on the other hand, Eq. (A-9) has terms which depend on the sign of $l$. This appears to result in the asymmetry of the solution with respect to the sign of $l$. In fact, the structures and energy budgets show a remarkable difference between DRY-POS and DRY-NEG wave (see Figs. 6 and 7). However, the growth rate looks symmetric with respect to $l=0$ (see Fig. 5). In the following, we will prove 
that the growth rate is in fact an even function of $l$ even in the non-geostrophic non-hydrostatic system. To this end, we introduce a new variable $R$ defined by

$$
w=\left(\frac{1-\varepsilon X}{1+\varepsilon X}\right)^{(i / 2)(l / k)} R .
$$

Then Eq. (A-9) becomes

$$
\begin{aligned}
\varepsilon X\left\{(\varepsilon X)^{2}-1\right\} \frac{d^{2} R}{d X^{2}}+2 \varepsilon \frac{d R}{d X} \\
+\varepsilon X\left\{\left(1+\frac{l^{2}}{k^{2}}\right)-\frac{l^{2}}{k^{2}} \frac{\varepsilon^{2}}{1-(\varepsilon X)^{2}}\right. \\
\left.-\delta^{-2}\left(1+\frac{l^{2}}{k^{2}}\right)(\varepsilon X)^{2}\right\} R=0,
\end{aligned}
$$

with the boundary conditions $R=0$ at $X=$ $-\sigma-\frac{k}{2}$ and $-\sigma+\frac{k}{2}$. Equation (A-11) and the boundary conditions do not depend on the sign of $l$, so that any set of $R$ and $X$ that satisfies Eq. (A-11) for a certain meridional wave number $l$ also satisfies the equation for the meridional wave number $-l$. Thus, the eigenvalue $\sigma$ is an even function of the meridional wavenumber $l$; i.e., the growth rate for the dry experiment is symmetric with respect to $l=0$. The structure and energy budgets for the dry experiment, however, is different between $l$ and $-l$ because the structure of $w$ depends on the sign of $l$ as seen in Eq. (A-10). This symmetry of the growth rate with respect to the sign of $l$ is not found for moist experiment (Fig. 2b) because the diabatic heating breaks the symmetry of Eq. (A-11).

\section{References}

Arakawa, A. and Moorthi, S., 1988: Baroclinic instability in vertically discrete systems, J. Atmos. Sci., 45, 1688-1707.

Craig, G. and Cho, H.R., 1988: Cumulus heating CISK in the extratropical atmosphere Part I: Polar lows and comma clouds, J. Atmos. Sci., 45, 2622-2640.

Eady, E.T., 1949: Long waves and cyclone waves, Tellus, 1, 33-52.

Gambo, K., 1976: The instability of medium-scale disturbances in a moist atmosphere, J. Meteor. Soc. Japan, 54, 191-207.

Harrold, T.W. and Browning, K.A., 1969: The polar low as a baroclinic disturbance, Quart. J. Roy. Meteor. Soc., 95, 710-723.
Hewson, T.D., Craig, G.C. and Claud, C., 2000: Evolution and mesoscale structure of a polar low outbreak, Quart. J. Roy. Meteor. Soc., 126, 1031-1063.

Mak, M., 1982: On moist quasi-geostrophic baroclinic instability, J. Atmos. Sci., 39, 20282037. , 1994: Cyclogenesis in a conditionally unstable moist baroclinic atmosphere, Tellus, 46A, 14-33.

Matsumoto, S., Yoshizumi, S. and Takeuchi, M., 1970: On the structure of the "Baiu Front" and the associated intermediate-scale disturbances in the lower atmosphere, J. Meteor. Soc. Japan, 48, 479-491.

Moorthi, S. and Arakawa, A., 1985: Baroclinic instability with cumulus heating, J. Atmos. Sci., 42, 2007-2031.

Ninomiya, K. and Akiyama, T., 1971: The development of the medium-scale disturbance in the Baiu front, J. Meteor. Soc. Japan, 49, 663677.

Rasmussen, E., 1979: The polar low as an extratropical CISK disturbance, Quart. J. Roy. Meteor. Soc., 105, 531-549.

— 1985: A case study of a polar low development over the Barents Sea, Tellus, 37A, 407418.

Reed, R.J. and Duncan, C.N., 1987: Baroclinic instability as a mechanism for the serial development of polar lows: a case study, Tellus, 39A, 376-384.

Romine, G. and Wilhelmson, R., 2002: A highresolution numerical simulation of the landfall of Hurricane Opal (1995), Bull. Amer. Meteor. Soc., 83, 1273.

Sardie, J.M. and Warner, T.T., 1983: On the mechanism for the development of polar lows, $J$. Atmos. Sci., 40, 869-881.

Stone, P.H., 1966: On non-geostrophic baroclinic stability, J. Atmos. Sci., 23, 390-400.

Tokioka, T., 1970: Non-geostrophic and nonhydrostatic stability of a baroclinic fluid, $J$. Meteor. Soc. Japan, 48, 503-520. , 1973: A stability study of medium-scale disturbances with inclusion of convective effects, J. Meteor. Soc. Japan, 51, 1-10.

Yanase, W., Niino, H. and Saito, K., 2002: High-resolution numerical simulation of a polar low, Geophys. Res. Lett., 29, 10.1029/ 2002 GL014736.

Yoshizumi, S., 1977: On the structure of intermediate-scale disturbances on the Baiu front, J. Meteor. Soc. Japan, 55, 107-120. 